\title{
ARTICLE
}

\section{Ultrastructural plasma membrane asymmetries in tension and curvature promote yeast cell fusion}

\author{
Olivia Muriel' ${ }^{1}$, Laetitia Michon¹, Wanda Kukulski² (D), and Sophie G. Martin ${ }^{1}$ D
}

\begin{abstract}
Cell-cell fusion is central for sexual reproduction, and generally involves gametes of different shapes and sizes. In walled fission yeast Schizosaccharomyces pombe, the fusion of $h+$ and $h$ - isogametes requires the fusion focus, an actin structure that concentrates glucanase-containing vesicles for cell wall digestion. Here, we present a quantitative correlative light and electron microscopy (CLEM) tomographic dataset of the fusion site, which reveals the fusion focus ultrastructure. Unexpectedly, gametes show marked asymmetries: a taut, convex plasma membrane of $h$-cells progressively protrudes into a more slack, wavy plasma membrane of $h+$ cells. Asymmetries are relaxed upon fusion, with observations of ramified fusion pores. $h+$ cells have a higher exo-/endocytosis ratio than $h$ - cells, and local reduction in exocytosis strongly diminishes membrane waviness. Reciprocally, turgor pressure reduction specifically in $h$-cells impedes their protrusions into $h+$ cells and delays cell fusion. We hypothesize that asymmetric membrane conformations, due to differential turgor pressure and exocytosis/endocytosis ratios between mating types, favor cell-cell fusion.
\end{abstract}

\section{Introduction}

Cell-cell fusion is a fundamental process underlying sexual reproduction and development. Plasma membrane (PM) merging for cell fusion requires a number of preparatory steps. These include the formation of cell adhesion, the remodeling of extracellular material to allow membrane contact, and significant force to overcome repulsive membrane and hydration charges. The actin cytoskeleton can generate this force and allow the engagement of membrane fusogenic machineries (Chan et al., 2020; Shilagardi et al., 2013). Fusogenic proteins, only identified in some organisms and cell types, then likely drive the formation of fusion pore(s) (Brukman et al., 2019; Hernández and Podbilewicz, 2017). However, fusion pore formation and expansion during cell-cell fusion have not been directly observed in any system.

Though fusion processes and machineries are extraordinarily diverse, the two cells engaging in fusion are asymmetric in the vast majority of cases. Fertilization largely happens between gametes of widely differing shape and size. Even Chlamydomonas reinhardi gametes, with very similar size and shape, show distinct mating structures, only one of them extending an actin-rich protrusion (Goodenough et al., 1982). The fusion of somatic cells also exhibits asymmetries, for instance during muscle development in Drosophila, where actin assembly in the myoblast powers podosome protrusion into the myotube, which responds through a myosin II-dependent cortical tension increase to promote fusion
(Kim and Chen, 2019; Kim et al., 2015; Sens et al., 2010). Similar asymmetries have been described in other types of somatic cell fusion (Brukman et al., 2019). By contrast, cell fusion during sexual reproduction in yeast is thought to happen between two isogametes, i.e., gametes of similar morphology.

The differences between yeast gametes are defined by the genetic information in the active mating type locus, which encodes distinct pheromone and receptor genes. Pheromone-dependent communication underlies sexual differentiation and the formation of cell pairs (Merlini et al., 2013). Fission yeast cells are naturally homothallic, i.e., they can switch between mating types, making the population mating-competent when faced with nitrogen starvation. Cells can also be fixed in one or other heterothallic mating type, $h-$ and $h+$, which can mate when mixed together. The two pheromones have distinct biochemical qualities and modes of secretion, and also exhibit slightly different functions and evolutionary constraints, suggesting some functional diversification between mating types (Merlini et al., 2013; Seike et al., 2019a; Seike et al., 2019b). However, very few additional genes are differentially expressed (Mata and Bähler, 2006; Xue-Franzén et al., 2006), and the cell fusion process itself is thought to happen between two symmetric cells.

The cell wall (CW) encasing yeast cells needs to be remodeled for cell morphogenesis. For cell growth, remodeling happens at

ínepartment of Fundamental Microbiology, University of Lausanne, Lausanne, Switzerland; ${ }^{2}$ Medical Research Council Laboratory of Molecular Biology, Cambridge, UK.

Correspondence to Sophie G. Martin: sophie.martin@unil.ch; W. Kukulski's present address is Institute of Biochemistry and Molecular Medicine, University of Bern, Bern, Switzerland.

(c) 2021 Muriel et al. This article is distributed under the terms of an Attribution-Noncommercial-Share Alike-No Mirror Sites license for the first six months after the publication date (see http://www.rupress.org/terms/). After six months it is available under a Creative Commons License (Attribution-Noncommercial-Share Alike 4.0 International license, as described at https://creativecommons.org/licenses/by-nc-sa/4.0/). 
the cell projection (also known as shmoo) tip, with turgor pressure providing the critical force (Atilgan et al., 2015; Minc et al., 2009). For cell fusion, the CW is locally digested to allow membrane contact. This requires an actin-based structure called the fusion focus, assembled by the formin Fusl, which serves to concentrate the myosin V-dependent delivery of secretory vesicles containing hydrolytic enzymes (Dudin et al., 2015). Turgor pressure is hypothesized to then help bring membranes in contact (Martin, 2016). Interestingly, we had previously described an asymmetry between mating types in the formation of the fusion focus, which is stabilized earlier in $h$ - than $h$ + cells (Dudin et al., 2015). While advanced live-cell imaging studies have revealed significant molecular and mechanistic information on the assembly of the fusion focus (Billault-Chaumartin and Martin, 2019; Dudin et al., 2015; Dudin et al., 2017; Dudin et al., 2016; Merlini et al., 2018), how the two cells shape their PMs for merging and how the initial fusion pore expands are not understood.

Here, we used correlative light microscopy (LM) and electron tomography to study cell fusion in Schizosaccharomyces pombe at the ultrastructural level. Our data show the organization of the fusion focus as a vesicle-rich structure that excludes other organelles. We describe all fusion stages, including pairs with membrane contact, and small and large fusion pores, indicating inhomogeneous pore expansion. Unexpectedly, the two prefusion gametes exhibit asymmetries in membrane tension and curvature, with $h$ - cells protruding into $h+$ cells. We trace the origin of these asymmetries to different ratios of endo- and exocytic activities and different turgor pressure between mating types, respectively, and propose that these asymmetries promote efficient cell-cell fusion.

\section{Results}

\section{Ultrastructure of the yeast fusion site}

We used correlative light and electron microscopy (CLEM) to study the ultrastructure of the actin fusion focus in homothallic (h90) WT strains expressing Fus1-sfGFP and Myo52-tdTomato. Using LM, we selected cell pairs exhibiting a Fus1 and Myo52 fusion focus signal, which allowed restriction of our analysis between $\sim 1 \mathrm{~h}$ before and $15 \mathrm{~min}$ after cell fusion, corresponding to the fusion focus lifetime (Dudin et al., 2015). We subsequently identified these pairs in transmission EM (TEM) and acquired tilt series to generate a dataset of 124 3D tomographic reconstructions (Fig. S1 A). Based on the minimal distance between the two PMs, we assigned each pair to one of four stages along the fusion process (Fig. 1, A-D). We distinguish three prefusion stages: two early stages where cells are in contact through their CW, with average minimal PM distance of $142 \pm 50$ $\mathrm{nm}$ for the first stage (far CW contact; 13 tomograms; Fig. $1 \mathrm{~A}$ ), and $49 \pm 26 \mathrm{~nm}$ for the second (close CW contact; 50 tomograms; Fig. $1 \mathrm{~B}$ and Video 1), where CWs have merged; and a third stage (PM contact; 11 tomograms; Fig. $1 \mathrm{C}$ ), with an average minimal PM distance of $5 \pm 4 \mathrm{~nm}$, where some regions of CWs are fully digested and the PMs appear in contact. The fourth stage is after fusion, where both cytosols are continuous. Among post-fusion pairs, the vast majority showed a single large fusion pore (43 tomograms; Fig. 1 D and Video 2). A further seven tomograms, difficult to classify, showed local loss of density in the CW suggestive of continuity between partner cells, and are described in more detail below. The distribution of tomograms according to minimal PM distances is shown in Fig. $1 \mathrm{E}$. While the relative frequency of tomograms in the close CW contact, $\mathrm{PM}$ contact, and small pore connections may be taken as time proxy for the progression of the fusion process, the earliest (far CW contact) and latest stages (large fusion pore) are underestimated, as we did not systematically acquire all tomograms for these stages, which could be readily identified from an initial TEM image at high magnification. Expansion of the fusion pore also likely progresses further after disassembly of the fusion focus.

Fusion foci appeared as accumulations of dense vesicles, as expected from the localization of Myo52 and secretory vesicle markers in LM images (Billault-Chaumartin and Martin, 2019; Dudin et al., 2015). We analyzed the density and size of the vesicles from 61 randomly chosen tomograms in our dataset at the cells' contact (see Materials and methods). Vesicle density did not change along stages (Fig. $1 \mathrm{~F}$ ) with an average of $0.65 \pm$ 0.22 vesicles $/ 10^{6} \mathrm{~nm}^{3}$. Vesicle size measurements revealed two populations: the dense vesicles mentioned above with an average diameter of $\sim 60 \mathrm{~nm}$, and a less abundant population with an average diameter of $\sim 30 \mathrm{~nm}$. The larger vesicles are likely secretory (as confirmed below), whereas the smaller ones may be endocytic, as dimensions are similar to previous measurements in Saccharomyces cerevisiae (Fig. 1 G; Kukulski et al., 2012a). Both vesicle populations were present throughout the different stages, with a somewhat lower frequency of the large vesicles in early stages (Fig. S1 B). Fusion foci also showed linear structures, likely representing actin filaments, predicted from the activity of the formin Fusl, which colocalized with Myo52 in LM (Fig. 2, Video 1, and Video 2). While only 51\% of tomograms revealed linear filament, which likely reflects the difficulty in preserving and detecting actin structures with this technique, the best-quality ones showed a large number of filaments positioned between vesicles and close to the PM. Actin filaments were observed in preand post-fusion cell pairs and could reach lengths $>0.5 \mu \mathrm{m}$. Fusion foci were largely devoid of ribosomes and other organelles, though these were present in other parts of the tomograms (Fig. 1, A-D; and Fig. 2). Microtubules were rarely observed prefusion (12\% cells), and more frequently after fusion ( $27 \%$ cells; Fig. S1 C). Interestingly, $52 \%$ cells showed organelles organized in sheets or reticulated with similar dense appearance as secretory vesicles (Fig. S1 D). Though we have not confirmed their identity, this is suggestive of Golgi or endosomal compartments, which may serve for local vesicle production. We conclude that fusion foci are dense vesicle- and actin-rich compartments that cannot easily be penetrated by other large cellular components.

\section{Shape of the fusion pore(s)}

To better understand the membrane merging process, we examined the shape of fusion pores. We identified a single cell pair with an apparent small pore (of diameter $\sim 45 \mathrm{~nm}$ ) entirely enclosed in the section (Fig. $3 \mathrm{~A}$ ). A filament can be seen traversing the pore. Seven tomograms showed a local loss of density in the CW at the interface between cells, suggestive of continuity between the two cells' cytosols (Fig. 3 B). However, the inner and 

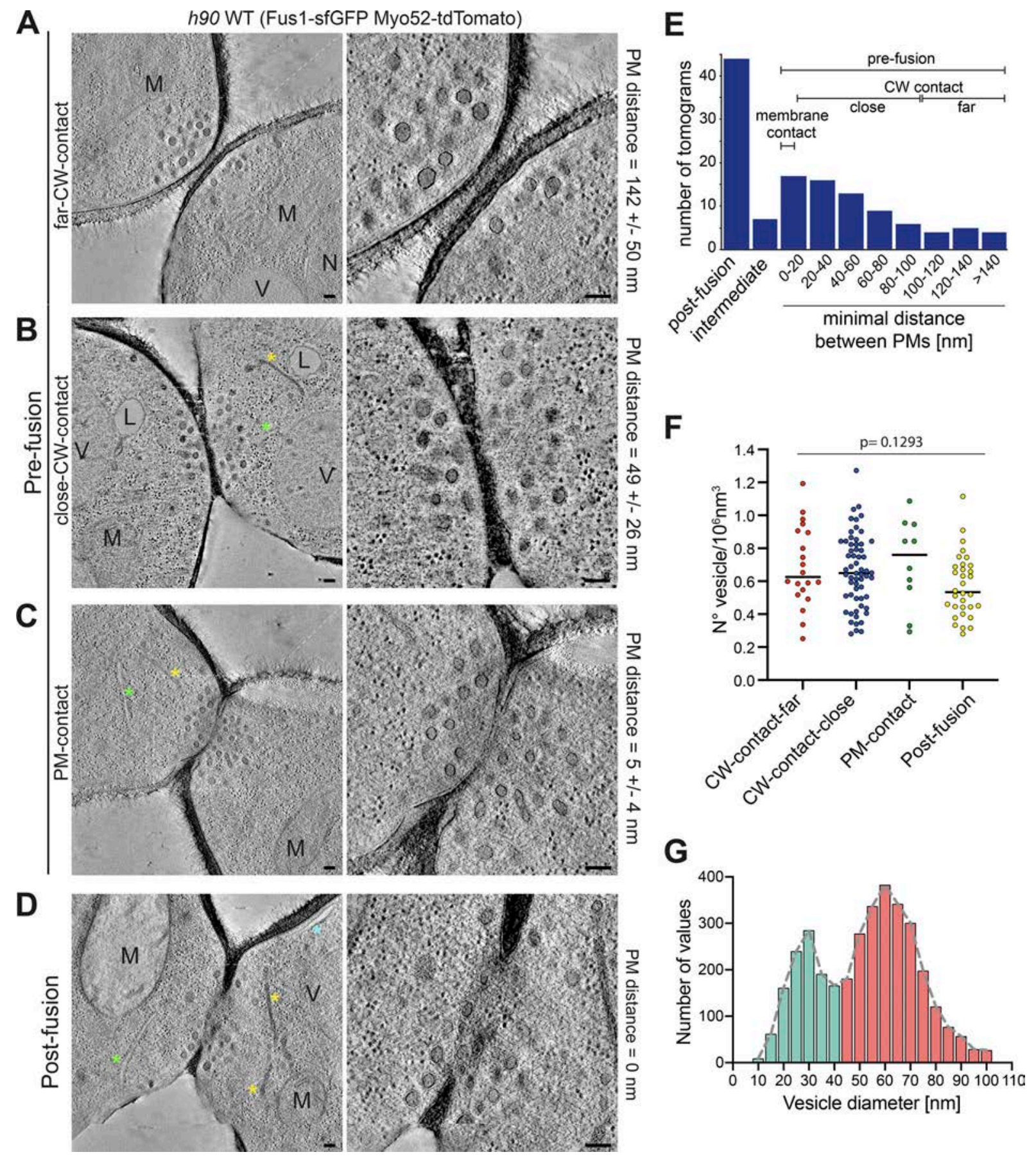

Figure 1. Correlative light-electron analysis of the yeast fusion site. (A-D) Virtual z-slices through tomograms of cell pairs during mating as identified by LM (see Fig. S1). Examples of different stages: far CW contact (A), close CW contact (B), PM contact (C), and after fusion (D). (E) Tomogram distribution according to minimal distance between PMs. (F) Vesicle density ( $n=20$ cells in far CW contact; $n=60 \mathrm{CW}$ contact close; $n=10$ PM contact; $n=32$ after fusion, including 8 with local loss of CW density). $P=0.1293$ (one-way ANOVA test). (G) Vesicle size distribution in cells as in $F$ ( $n=122$ cells and 3,521 vesicles). Bin width $=5 \mathrm{~nm}$. Blue asterisk = cortical smooth ER; green asterisk = rough ER; yellow asterisk = dense sheet or reticulated organelle; $M=$ mitochondrion; $V=$ vacuole; $\mathrm{L}=$ lipid droplet; $\mathrm{N}=$ nucleus. Scale bars, $100 \mathrm{~nm}$.

outer membrane leaflet could not be unambiguously resolved from the CW density to conclusively distinguish between local CW digestion and PM contact, or possible fusion intermediates or even fully formed tiny pores. In three cases, these connections between partner cells were entirely included in the tomographic volume. In the four other cases, they extended to one or both section edges, with one tomogram displaying several such connections, suggesting that membrane merging can initiate at multiple positions (Fig. 3 C, Fig. S2 A, and Video 3). However, as these connections extend to the edge of the tomographic volume, we cannot know with certainty whether they initiated independently or represent extensions of a single opening. 
A
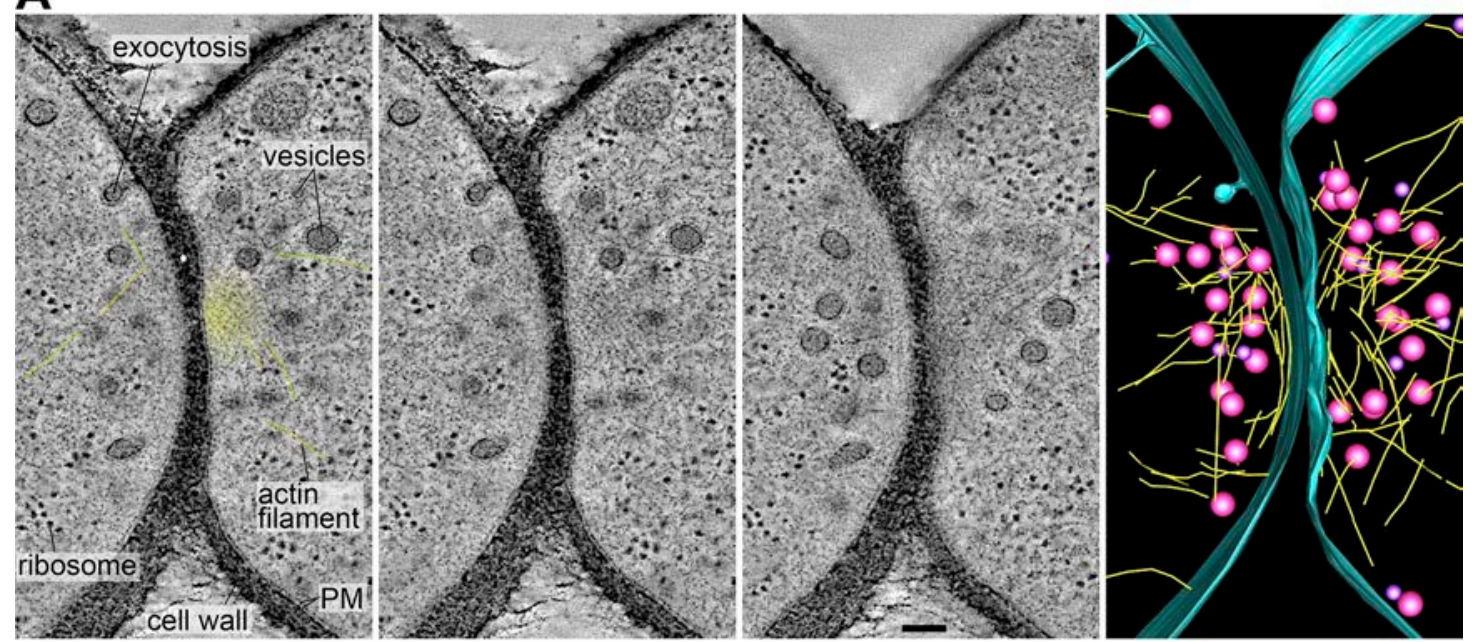

B
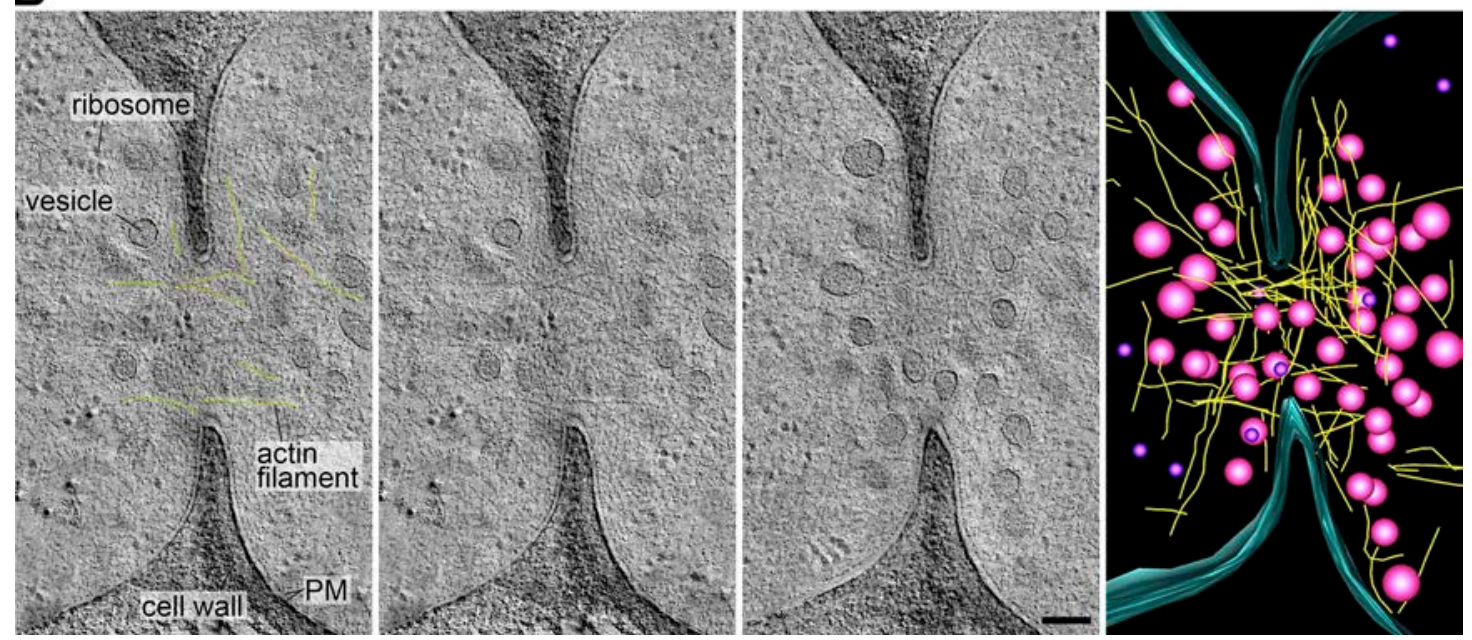

Figure 2. Actin fusion focus ultrastructure. (A and B) Virtual z-slices through tomograms and model of the fusion focus including vesicles (magenta), actin filaments (yellow), and the PM outer leaflet (blue) in cell pairs before (A) and after fusion (B). The first two images are identical, with and without labels. Yellow lines overlay the clearest actin filaments. The yellow haze in the top pair highlights a region likely dense with actin filaments that cannot be individually resolved.. Scale bars, $100 \mathrm{~nm}$.

Among pairs with a larger fusion pore, two classes retained our attention, as they exhibited nonspherical pore edges. Eight tomograms showed pore edges that extended into irregular extensions, such that at some cross-section of the tomographic volume, they appeared as the fusion intermediates described above (Fig. 3 D; Fig. S2, B and D; Video 4; and Video 5). Six further tomograms showed a large pore that split into two openings separated by a strand of CW (Fig. 3 E, Fig. S2 C, and Video 6). These observations suggest two non-mutually exclusive interpretations. First, initial fusion may occur at several locations, forming several small pores that fuse together to form a nonspherical larger pore (Fig. 3 F, i). Second, fusion may initiate at a single location, but pore expansion is limited by CW degradation and occurs nonhomogeneously, leading to fingerlike extensions of the pore periphery (Fig. $3 \mathrm{~F}$, ii). In either case, the small number of tomograms showing PM contact and small pores suggests that membrane fusion and initial pore expansion are rapid relative to $\mathrm{CW}$ digestion.

\section{PM shape changes during the fusion process}

To learn more about possible biophysical changes for PM merging, we focused our attention on the conformation of the PM in prefusion cell pairs. While yeast gametes are considered isogametes, we identified two morphological asymmetries at the ultrastructural level. First, the PM at the shmoo tip exhibited different levels of tension: its appearance ranged from taut, forming a smooth arc along the CW (smooth PM [sPM]), to slack/floppy, forming waves (wavy PM [wPM]). In most prefusion pairs, one cell had wPM and the other sPM (60\%; 44/74), but other configurations ( $35 \%$ or $26 / 74$ with both cells sPM; $5 \%$ or 4/74 with both wPM) were also observed (Fig. 4, A-C). The frequency of PM waviness, whether in one or both partner cells, increased along the fusion process and decreased after fusion (Fig. 4 D). Indeed, most post-fusion pairs showed sPM (84\%; 36/ 43; Fig. S3 A).

Second, we found that the curvature of the zone of cell-cell contact varied, ranging from convex (positive curvature) to flat 
A
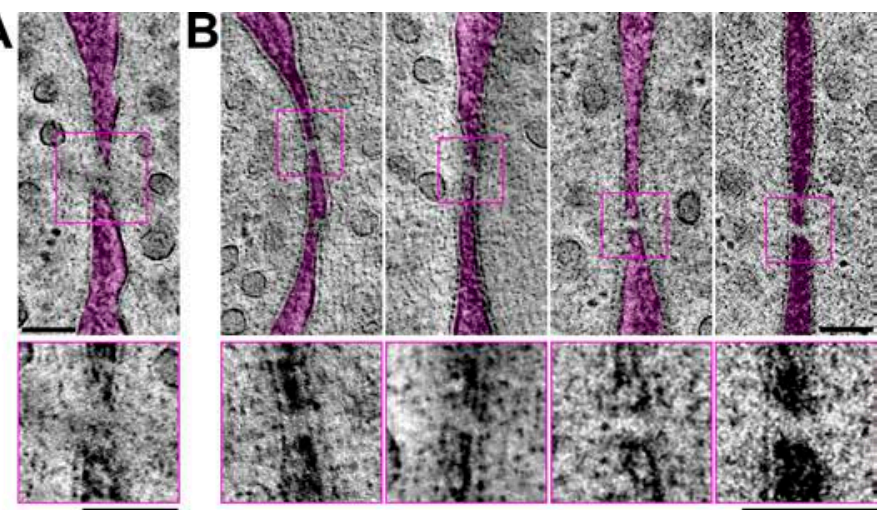

C

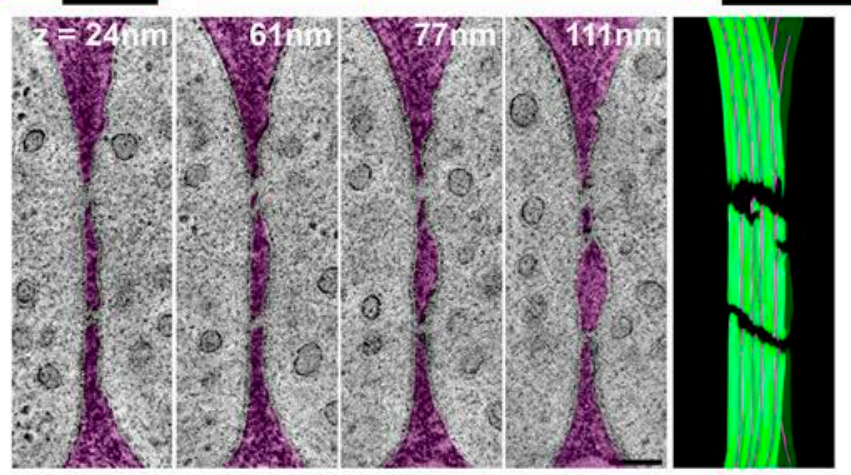

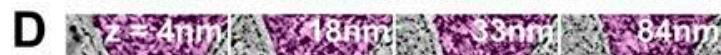

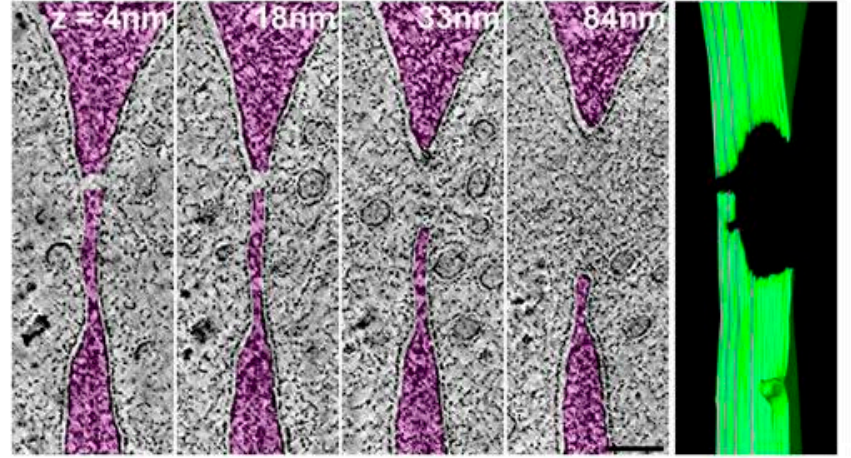

E

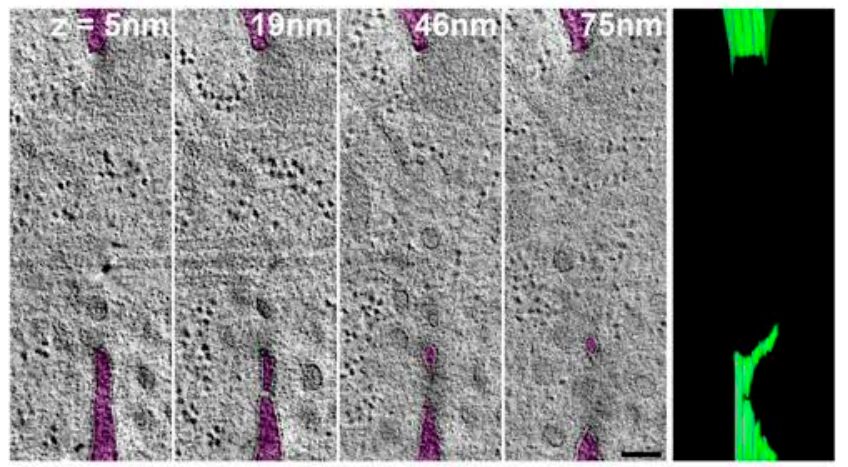

$\mathbf{F}$

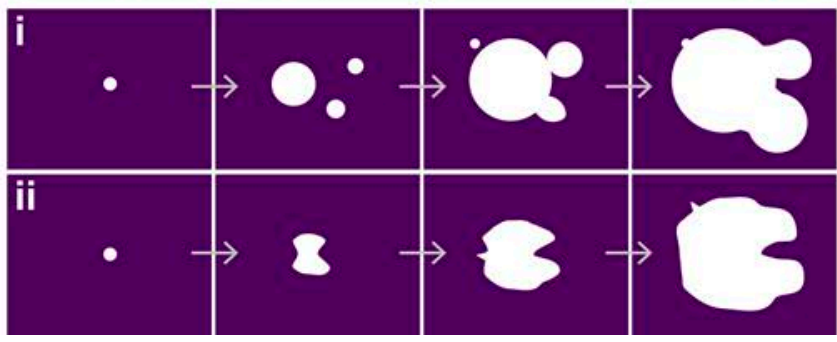

Figure 3. Shape of the fusion pore. (A-E) Virtual z-slices through tomograms of cell pairs, showing one small pore entirely enclosed in the tomographic volume (A) and four cases of apparent connection between cells, with local loss of density in the CW, some of which may represent fusion intermediates (B). The CW is colored in purple. Zoomed views are shown at the bottom. (C-E) Four virtual z-slices through tomograms and model of the PM outer leaflet. Z-slice positions from left to right are indicated by purple lines on the model, where the $z$-slice intersects the green PM outline at a perpendicular angle (see Video 3, Video 4, and Video 6). (C) Several connections, similar to the ones shown in B. (D and E) Large pores with an irregular edge. $\ln D$, the larger pore at $z=84 \mathrm{~nm}$ is prolonged into a connection as in $B$ at $z=18$ and $4 \mathrm{~nm}$. In $\mathrm{E}$, a large pore at $z=5 \mathrm{~nm}$ splits into two openings separated by a strand of CW at higher $z$ levels. The $\mathrm{CW}$ is colored in purple. Uncolored images are shown in Fig. S2, A-C. (F) Two non-mutually exclusive interpretations of fusion pore formation: fusion may initiate at several locations, forming pores that fuse together to form a nonspherical larger pore (i); fusion may initiate at a single location, but pore expansion occurs nonhomogeneously, limited by CW degradation (ii). Scale bars, $100 \mathrm{~nm}$. 

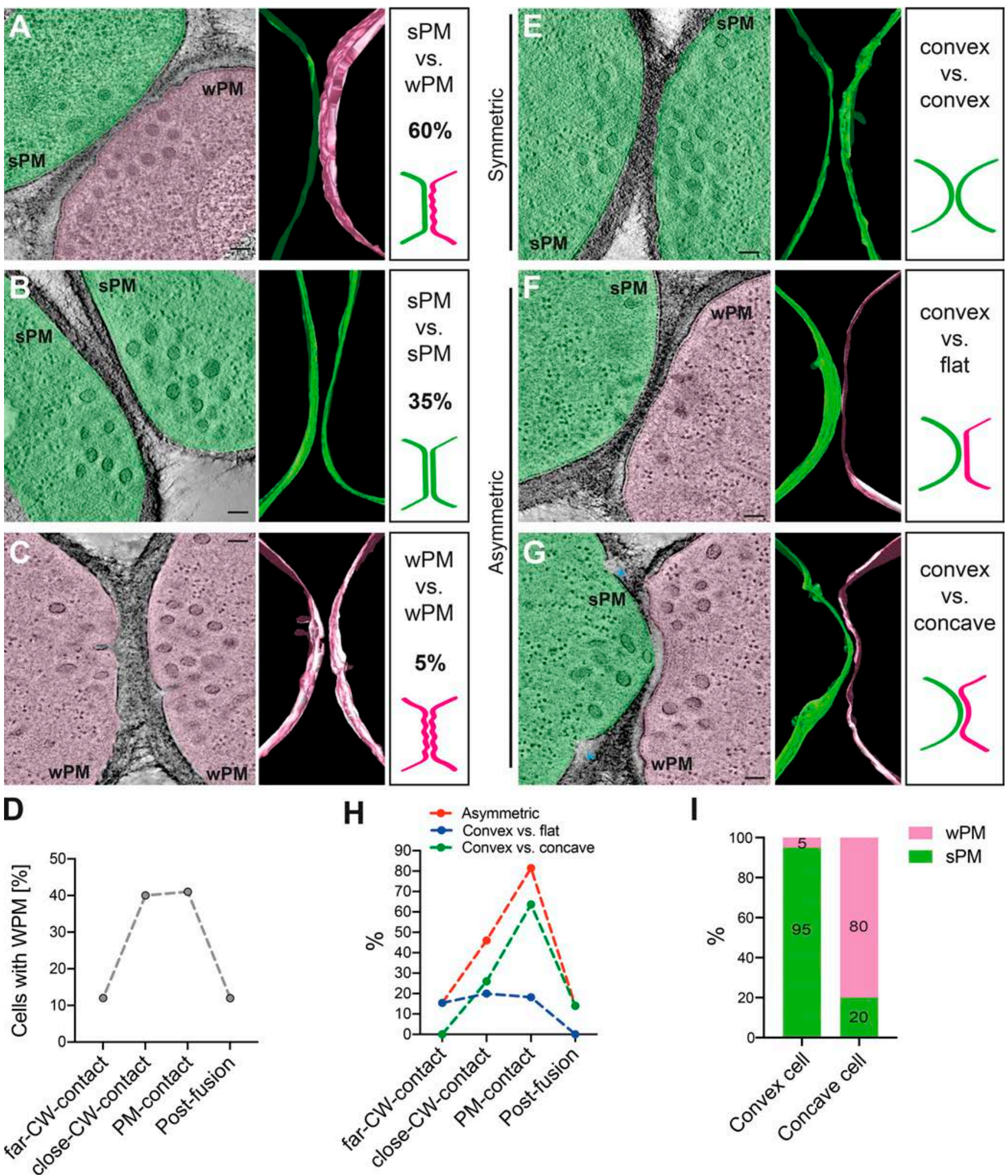

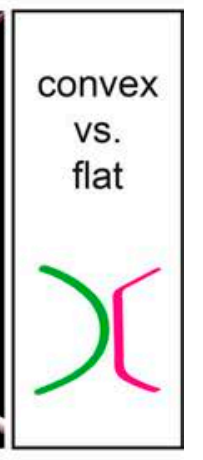

convex

vs. concave
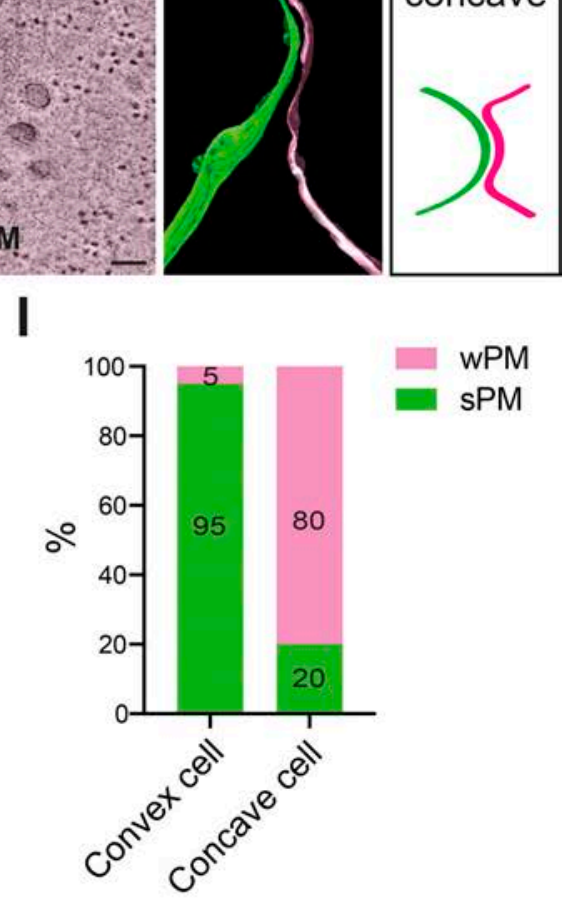

Figure 4. PM shape changes in preparation for fusion. (A-C) Virtual z-slices through representative tomograms of mating cells showing sPM versus wPM (the predominant situation; A), sPM in both (B), and wPM in both cells (C). Segmentation models of the PM outer leaflet and schematic drawings are shown at the right. (D) Percentage of cells with wPM along fusion stages ( $n=26$ far CW contact cells; $n=100$ close CW contact; $n=22$ PM contact and $n=86$ after fusion). (E-G) Virtual slices through representative tomograms of mating cells showing symmetric (convex versus convex; E) and asymmetric PM curvatures (convex versus flat [F] and convex versus concave [G]). Segmentation models of the PM outer leaflet and schematic drawing are shown at the right. (H) Percentage of asymmetric mating pairs along fusion stages ( $n=13$ far CW contact pairs; $n=50$ close CW contact; $n=11$ PM contact and $n=43$ after fusion). (I) Percentage of cells with SPM or WPM in prefusion pairs showing a convex-concave configuration, showing that low PM tension is associated with the concave state. $n=20$ pairs. Scale bars, $100 \mathrm{~nm}$. 
to even concave (negative curvature). We called the situation symmetric when both partner cells showed similar positive or null curvature (Fig. $4 \mathrm{E}$ ), and asymmetric when the cells showed distinct curvature (Fig. 4, F and G). Asymmetry was observed in $46 \%(34 / 74)$ of prefusion cell pairs. In these pairs, one cell is convex while the other is either flat (Fig. $4 \mathrm{~F}$ ) or concave (Fig. $4 \mathrm{G}$ ), such that one cell protrudes into the other (see also Fig. S3). Several protruding cells also showed unresolved membrane invaginations on either side of the protrusion, of unknown origin (Fig. S3, B-D). The frequency of asymmetric pairs, especially convex versus concave, increased along the fusion process, with a maximum $>80 \%$ at the PM contact stage, and decreased after fusion (Fig. $4 \mathrm{H}$ ).

Finally, we found that PM waviness and overall curvature were highly correlated in prefusion cells: $80 \%$ of concave cells were wavy, and $95 \%$ of convex cells were smooth (Fig. 4, F, G, and I; and Fig. S3). Together, these data show that partner cells become highly asymmetric during the fusion process: one cell exhibits a taut SPM and protrudes into its partner, which exhibits a slack wPM. This asymmetry is relaxed after fusion.

\section{PM asymmetries are mating type-specific}

The asymmetry of prefusion partner cells suggests that each morphology may be associated with a specific mating type. To address this hypothesis, we repeated our CLEM-tomography approach using WT heterothallic strains $(h+$ and $h-)$, of which only one was labeled with mCherry-D4H, a PM-localized sterol biosensor (Marek et al., 2020), to identify mating type, and with Fusl-sfGFP to identify pairs in fusion. We generated 22 tomograms ( 15 with $h+$ labeled, 7 with $h$ - labeled) at the CW contact stage. As in the $h 90$ strain, we observed PM asymmetries in waviness and curvature: $73 \%$ of pairs $(16 / 22)$ showed sPM versus wPM, and 63\% (14/22) showed asymmetric curvature (Fig. S4 A). Importantly, cell morphology and mating type were correlated: $73 \%(16 / 22)$ of $h+$ cells, but only $18 \%(4 / 22)$ of $h$ - cells, had wPM (Fig. $5 \mathrm{~A}$ and Fig. S4 B). Furthermore, in pairs with asymmetric curvature, $79 \%$ (11/14) of the convex cells were $h-$ (Fig. 5 B and Fig. S4 C). We conclude that cell asymmetries are largely conditioned by mating type, in which the $h$ - cell protrudes into an $h+$ cell with wPM.

\section{Cell type-specific balance of exo- and endocytosis}

The taut versus floppy PM appearance in $h-$ and $h+$ cells suggests a possible change in the balance of exo- and endocytosis in the two cell types. We hypothesized that PM waves result from reduced endocytic and/or increased exocytic rates, leading to an "excess" of PM. To test this hypothesis, we first used LM to measure the relative levels of endo- and exocytic vesicle-associated proteins. The endocytic vesicle-associated Fimbrin (Fim1) in pairs labeled by a Myo52-tdTomato focus was sufficiently internal to unambiguously distinguish the signal from each cell. This allowed to compare Fiml-sfGFP levels in $h+$ and $h$ - partners at the same fusion stage, thus eliminating potential stagespecific variations. To distinguish mating types, $h+$ cells further expressed cytosolic mTag-BFP2. We observed a $33 \pm 12 \%$ reduction in Fiml-sfGFP levels in $h+$ cells (Fig. 5 C), indicating a reduced amount of endocytosis in $h+$ cells.
To estimate secretion, we used Myo52-tdTomato, which associates with secretory vesicles. Because the fluorescence signals from the two partner cells overlap, we analyzed Myo52tdTomato expressed in a single mating type crossed with the other, unlabeled mating type. We observed a roughly twofold increase in Myo52-tdTomato fluorescence in $h+$ cells (Fig. 5, D-F). These cells also expressed Fim1-sfGFP, which reproduced the reduced endocytic signal in $h+$ cells observed above, thus excluding artifacts due to stage-specific or experimental signal variation. Similar results were obtained using the secretory vesicle-associated exocyst subunit Exo84, which showed a 1.5 -fold signal increase in $h+$ cells (Fig. S4 D). Thus, $h+$ cells show higher levels of exocytic and lower levels of endocytic signal than $h$ - cells.

We reasoned that such a difference in vesicular trafficking between mating types should also be observed in the amount and size of vesicles detected by EM. Indeed, in the labeled $h+x$ $h$ - cell pairs, $h+$ cells showed a $30 \%$ higher vesicle density, with an increased number of 60-nm, secretory vesicles, while the 30$\mathrm{nm}$ vesicle population was slightly reduced compared with $h-$ cells (Fig. $5 \mathrm{G}$ ). In our larger $\mathrm{h} 90$ tomogram dataset, cell type identity is unknown, but may be inferred in pairs showing clear PM asymmetries, i.e., a convex cell with sPM (inferred to be $h-$ ) mating with a concave partner with wPM (inferred to be $h+$ ). In this analysis, we obtained an $23 \%$ increase in vesicle density in the concave/wPM, with a larger population of $60-\mathrm{nm}$, secretory vesicles and slight reduction in the $30-\mathrm{nm}$ population (Fig. $5 \mathrm{H}$ ). Together, this set of data suggests that the difference in PM waviness between $h+$ and $h$ - cells is a consequence of altered balance between endocytosis and exocytosis in the two cell types.

\section{PM waviness requires local exocytic activity}

To test the idea that membrane waviness is a consequence of excess exocytosis over endocytosis, we aimed to reduce local exocytosis. Deletion of Fusl, necessary for the assembly of the actin fusion focus, is predicted to prevent secretory vesicle accumulation, and thus reduce secretion locally. Because cell fusion completely fails when both partner cells lack fusl, we performed CLEM in heterothallic crosses between unlabeled fus $1 \Delta$ and WT cells expressing Fusl-sfGFP and mCherry-D4H, allowing identification of pairs in fusion and mating types, respectively ( $n=27$ tomograms for $h+$ fusl $\Delta$, Fig. 6 A, Fig. S4 E, and Fig. S5 A; $n=20$ tomograms for $h-$ fusl $\Delta$, Fig. 6 B, Fig. S4 E, and Fig. S5 B). The density of vesicles was strongly reduced in the fusl $\Delta$ cells, whether this was $h+$ or $h-$, while WT $h+$ cells showed slightly higher vesicle density than $h$ - cells, as in previous analysis (Fig. 6, A-C). The reduced vesicle density in fusis is due to a strong decrease of the $60-\mathrm{nm}$ vesicles, confirming that these are secretory (Fig. 6 D). We also searched for linear structures, which we presumed above to be actin filaments: in the 24 tomograms in which we could identify filaments in the WT cell, only eight fusls cells showed filaments near the cell-cell contact region. These results confirm at the ultrastructural level that Fus1 is necessary for formation of the actin fusion focus and the concentration of secretory vesicles. By LM, Myo52 and Exo84 also showed strong signal reduction in fusl $\Delta$ (Fig. $6 \mathrm{E}$ and Fig. S4 D). Curiously, the endocytic vesicle marker Fim1-sfGFP signal 


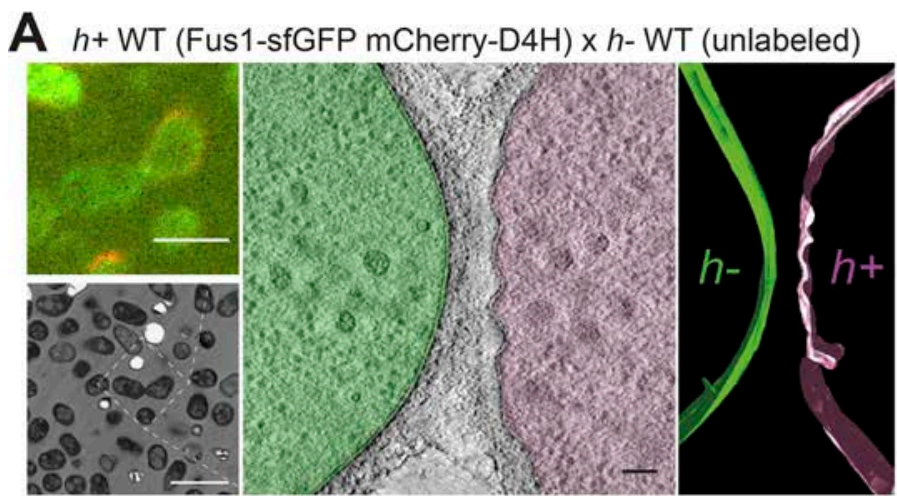

B

$h+$ WT (unlabelled) $\times h$-WT (Fus1-sfGFP mCherry-D4H)
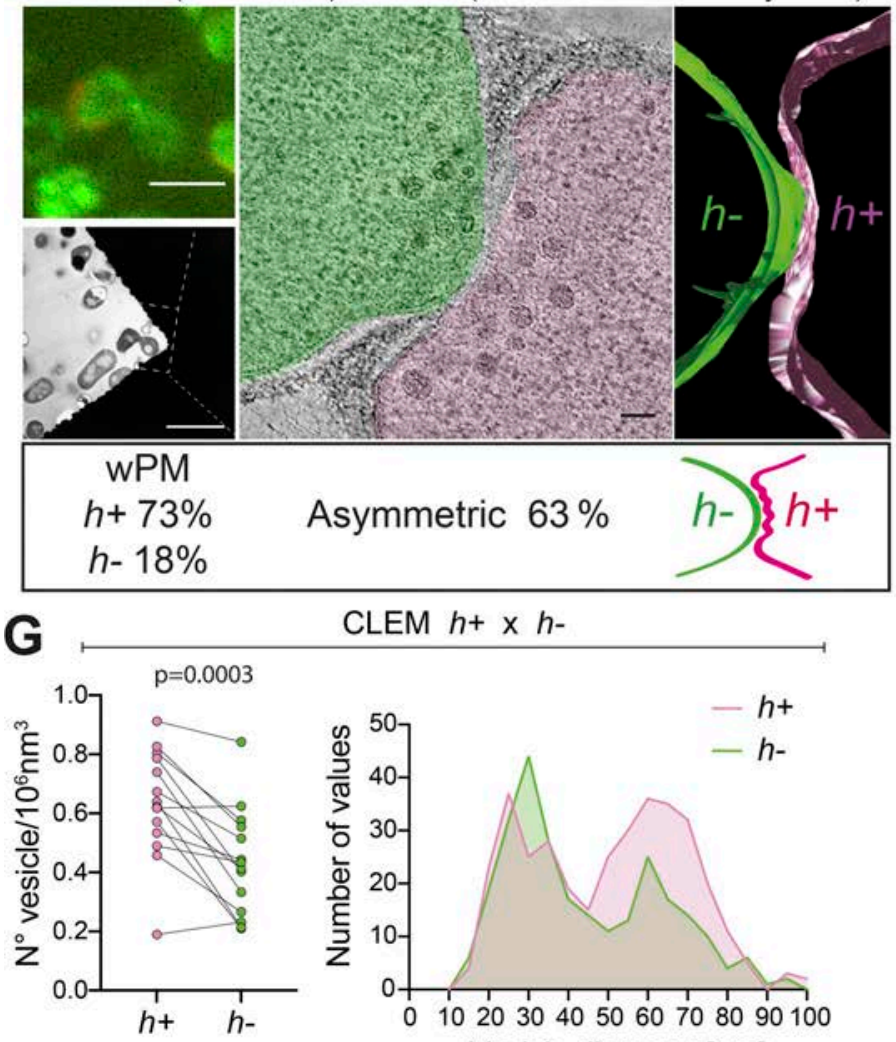

CLEM $h+\times h-$

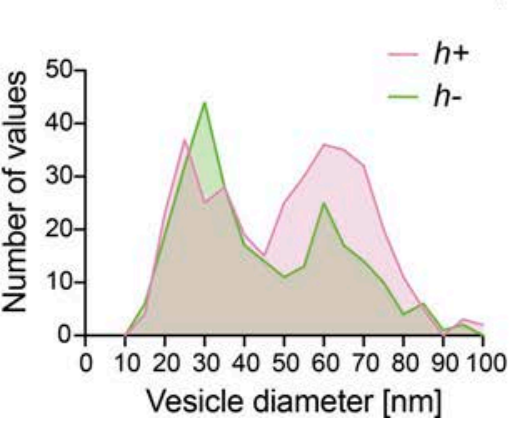

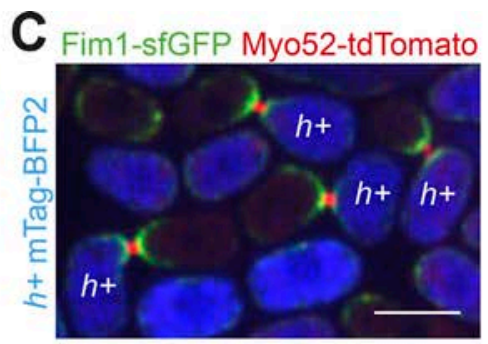
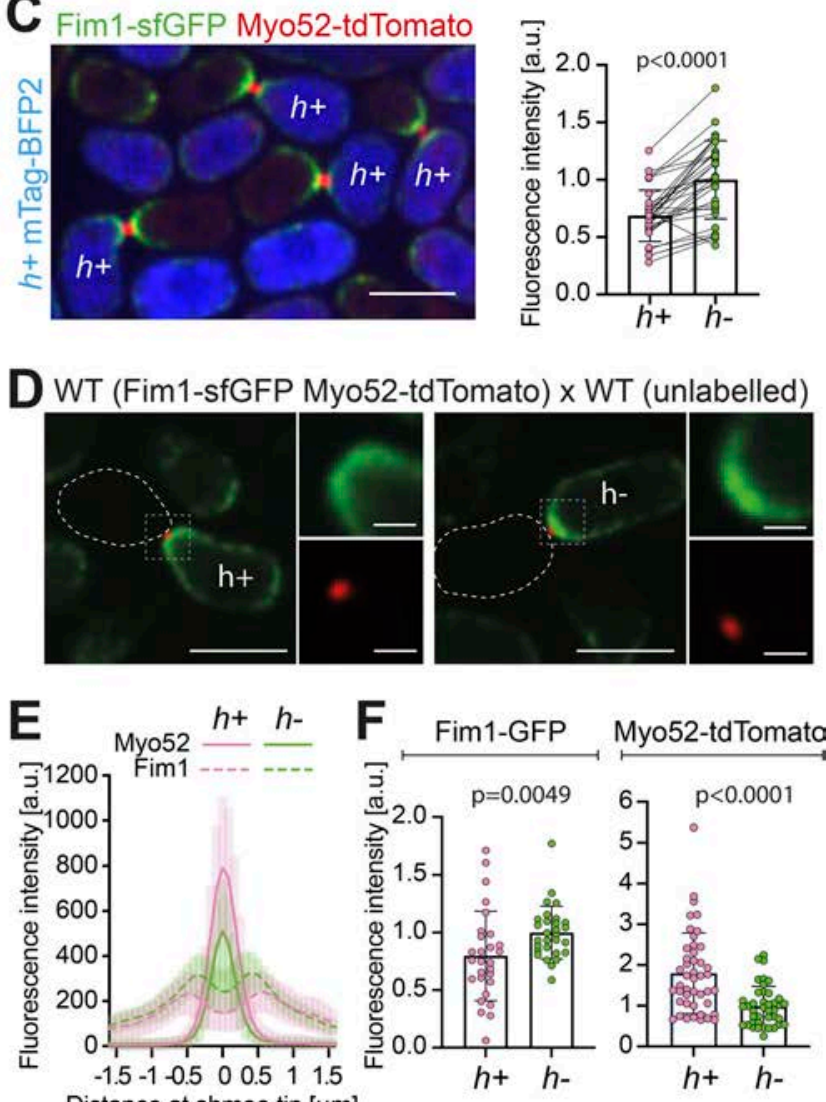

Distance at shmoo tip $[\mu \mathrm{m}]$

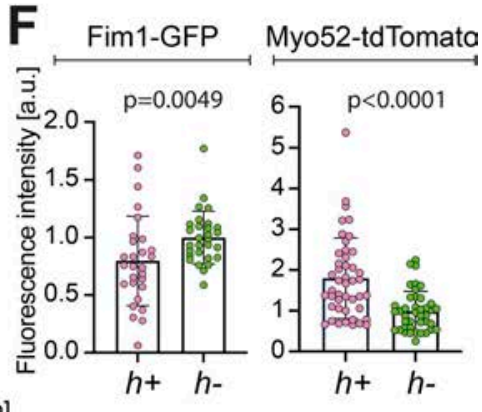

CLEM $h 90$
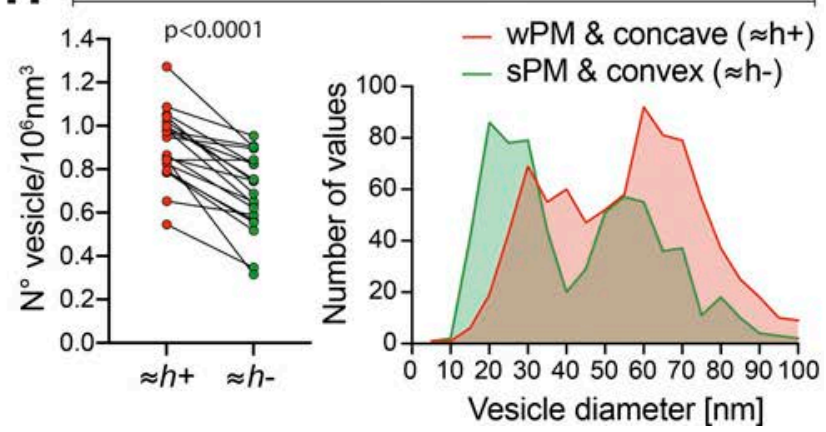

Figure 5. Mating type-specific PM asymmetries and vesicle distributions. (A and B) Representative examples of WT cell pairs with one mating type labeled showing WPM versus SPM (A), and convex versus concave PM curvature (B). Top left LM images show Fus1 in green and cell identity in red, based on mCherry-D4H expression. Bottom left TEM pictures show the same mating pair. A virtual z-slice of the tomogram shows PM shapes, segmented with Imod. (C) Average time-lapse projection of Fim1-sfGFP (green) and Myo52-tdTomato (red) expressed in both cells. Cytosolic mTag-BFP2 (blue) is expressed only in $h+$ cells. Paired Fim1 fluorescence intensities at the shmoo tips are shown on the right ( $n=31$ pairs in two experiments; $P<0.0001$; paired $t$ test). Individual data, mean \pm SD, are shown. (D) Average time-lapse projection of Fim1-sfGFP (green) and Myo52-tdTomato (red) present only in one cell type (left: $h+$; right: $h$-) mated with unlabeled partner. (E) Fluorescence profile of Fiml and Myo52 along the shmoo tips of cells as in D ( $n=13 h+$ and $13 h-$ cells). (F) Fiml and Myo52 mean fluorescence intensity of the 5 maximum pixels around each peak from profiles as in $E(n=28 h+$ and $29 h$ - cells from two experiments for Fiml and $n=$ $58 h+$ and $52 h$ - cells from four experiments for My052; P values from Mann-Whitney tests). (G) Vesicles paired density and size distribution in prefusion WT $h+/ h$ - cell pairs. $n=14$ tomograms (264 vesicles for $h-; 350$ for $h+$ ), $P=0.0003$; paired $t$ test. (H) Vesicle paired density and size distribution in prefusion WT h90 cells classified according to PM curvature and waviness. $n=20$ tomograms (669 vesicles for sPM/convex; 830 for wPM/concave cells), $P<0.0001 ;$ paired $t$ test. Scale bars are $5 \mu \mathrm{m}$ in LM ( $1 \mu \mathrm{m}$ for insets), $10 \mu \mathrm{m}$ in TEM, and $100 \mathrm{~nm}$ in tomogram slices.

was also slightly reduced in fusl $\Delta$ (Fig. 6 F). Neither Myo52 nor Fim1 levels were significantly different between mating types in fusis cells (Fig. 6, E and F). Thus, local secretion at the cell-cell contact site is decreased in fusl $\Delta$ cells, and this likely has consequences for endocytic activity.
Interestingly, the reduction in local secretion correlates with a strong loss of the wPM phenotype (Fig. 6, A and B; Fig. S4, E and F; and Fig. S5): only $4 \%$ of $h+$ fusl $\Delta$ cells ( $1 / 27)$ showed wPM, whereas $73 \%(16 / 22)$ and $20 \%(4 / 20)$ of $h+W T$ showed wPM in $\mathrm{WT} \times \mathrm{WT}$ and WT $\times$ fusis crosses, respectively. The frequency 

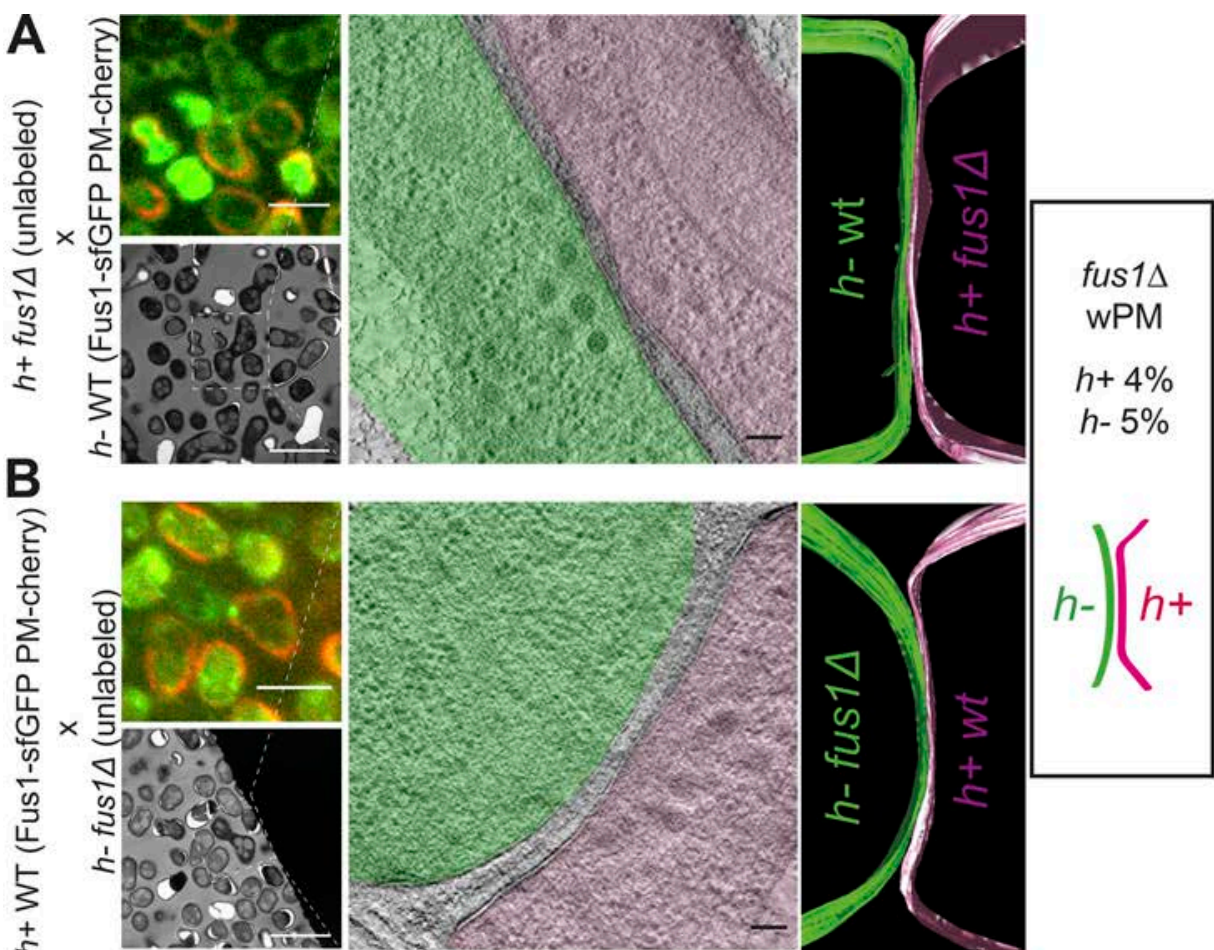

C
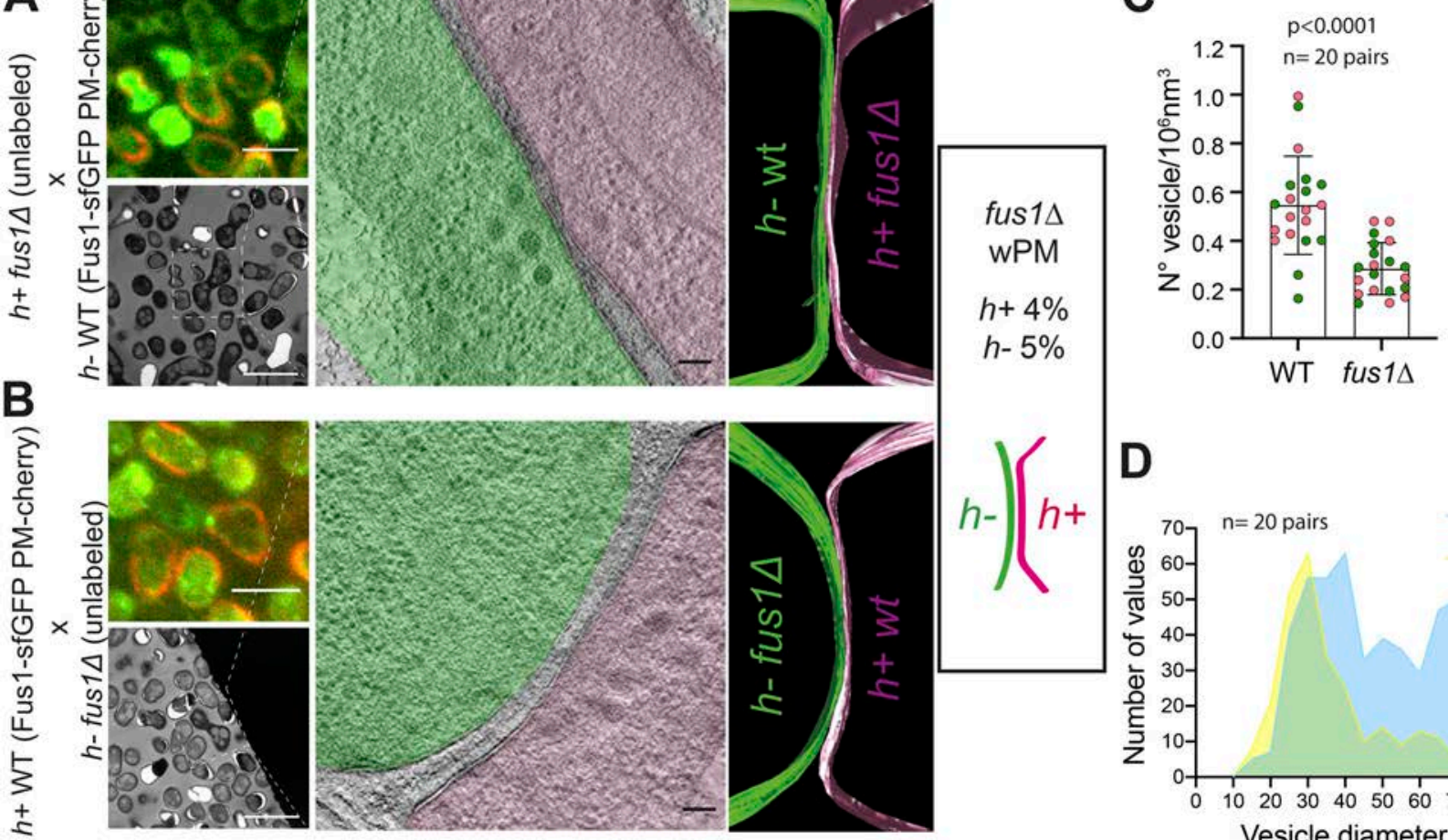

D
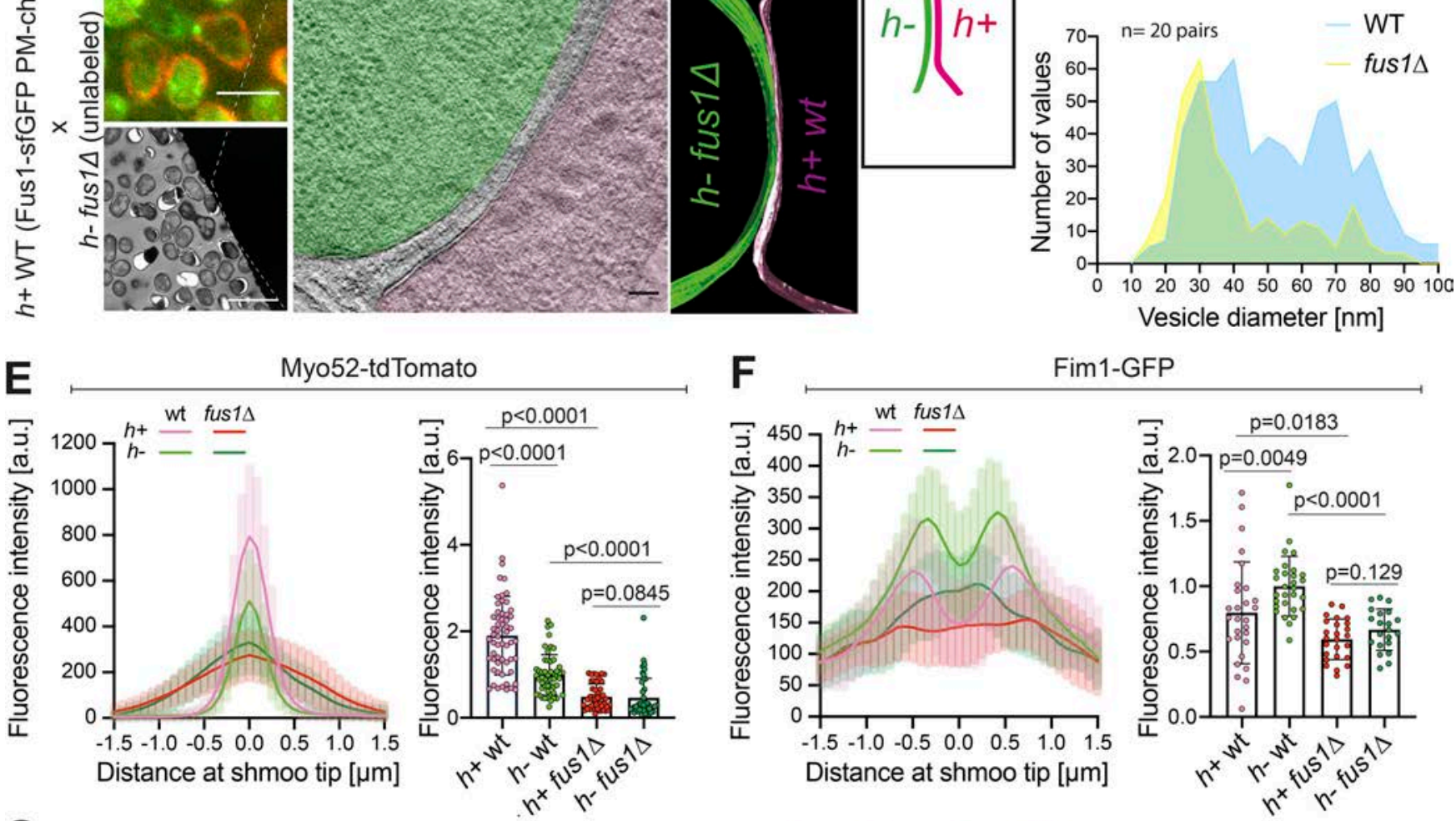

$\mathbf{F}$

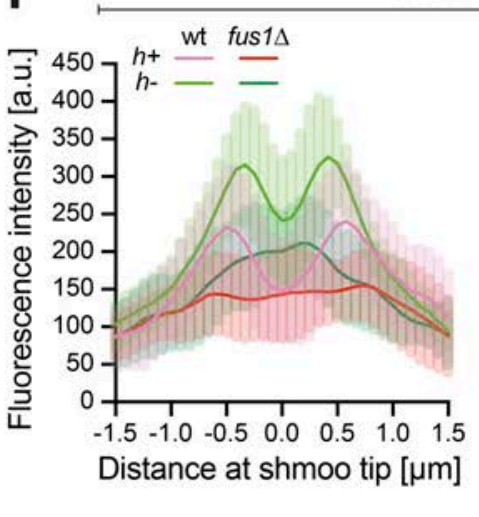

Fim1-GFP
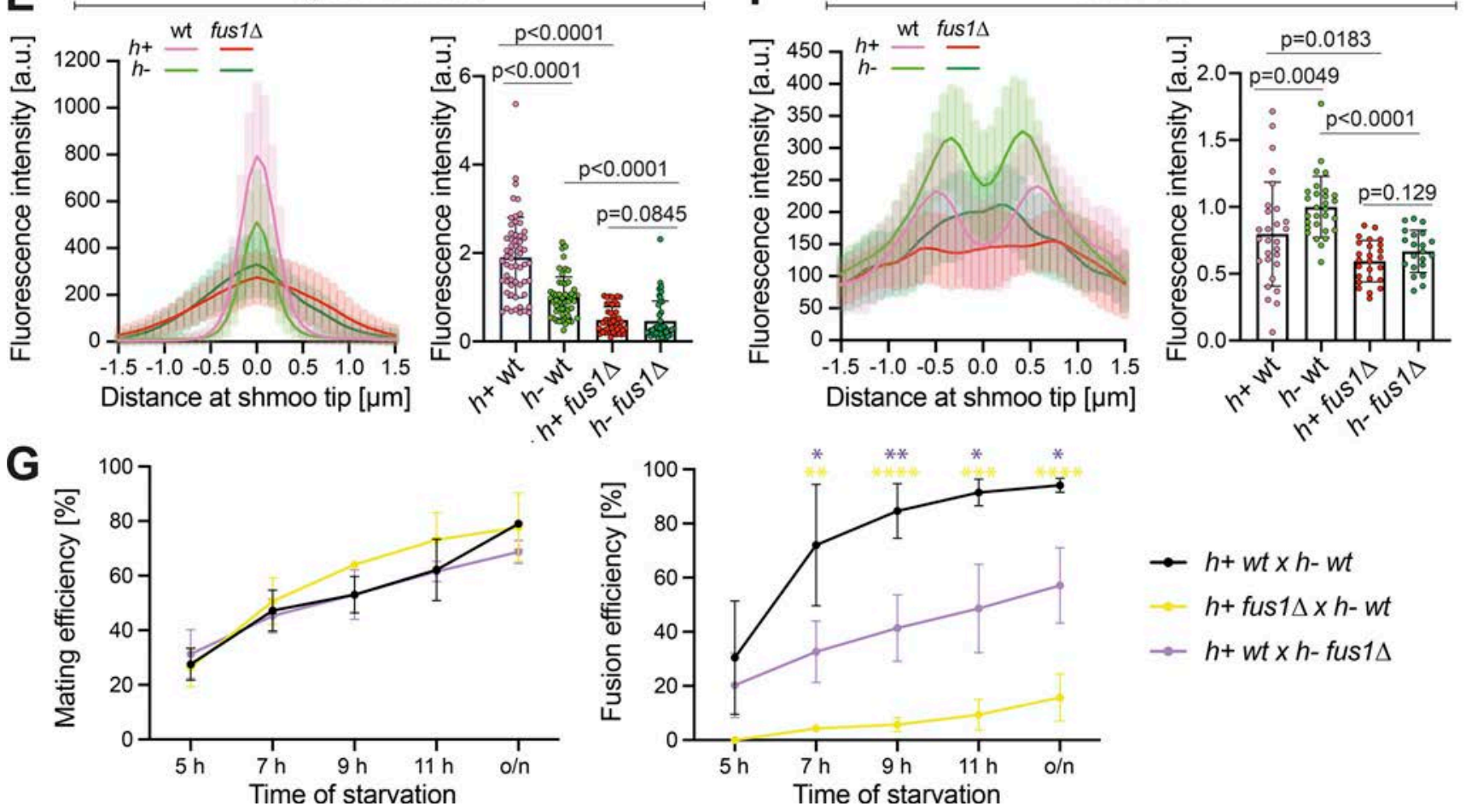

$\rightarrow h+w t x h-w t$

$-h+$ fus $1 \Delta x h-w t$

$\rightarrow h+w t \times h-f u s 1 \Delta$

Figure 6. Local exocytosis underlies PM waviness. (A and B) Representative example of WT crossed with fus1 cells. The top left LM pictures show Fus1sfGFP (green) and mCherry-D4H (red) expressed in the WT cell. Bottom left TEM pictures show the same mating pair. The virtual tomogram slices show PM shapes, segmented with Imod. Most fus1 $\Delta$ cells, whether $h+(A)$ or $h-(B)$, exhibit sPM. Scale bars are $5 \mu \mathrm{m}$ in LM, $10 \mu \mathrm{m}$ in TEM, and $100 \mathrm{~nm}$ in tomogram slices. (C) Vesicle density in WT and fus1 $\Delta$ prefusion cells. $n=40$ cells, $P<0.0001$, unpaired $t$ test. Magenta dots are $h+$ cells; green dots are $h-$ cells. (D) Vesicle size distribution from C ( $n=566$ vesicles for WT, 297 for fus $1 \Delta)$. Bin width $=5$. (E) Myo52-tdTomato average time-lapse projection fluorescence profiles along shmoo tips of $h+$ and $h-$ fus $1 \Delta$ cells crossed to unlabeled WT ( $n=13 h+W T, 13 h-W T, 12 h+f u s 1 \Delta, 9 h-$ fus $1 \Delta)$. Right: Myo52 mean fluorescence intensity of the 5 maximum pixels from profiles as on the left ( $n=51 \mathrm{~h}+$ fus $1 \Delta, 45 \mathrm{~h}$ - fus $1 \Delta$ cells from four experiments; P values are from Mann-Whitney tests). WT values are identical to Fig. 5, E and F. (F) Fim1-sfGFP average time-lapse projection fluorescence profiles along $h+$ and $h$ - fus $1 \Delta$ shmoo tips $(n=13 h+W T, 13 h-W T, 12 h+$ fus $1 \Delta, 9 \mathrm{~h}$ - fus1 $1 \Delta)$. Right: Fim1 mean fluorescence intensity of 5 maximum pixels from each peak of profiles as on the left $(n=28 \mathrm{~h}+\mathrm{WT}, 29 \mathrm{~h}-\mathrm{WT}, 25 \mathrm{~h}+$ fus $1 \Delta$, 
$21 \mathrm{~h}$ - fus $1 \Delta$ cells from two experiments; P values from Mann-Whitney tests). WT values are identical to Fig. 5, E and F. (G) Mating and fusion efficiencies over time ( $n \geq 500$ cells of each genotype from three to five experiments; bars show SD; ${ }^{*}, P<0.05 ;{ }^{* *}, P<0.01 ;{ }^{* * *}, P<0.005 ;{ }^{* * *}, P<0.0001$ from unpaired $t$ tests).

was similarly reduced in $h$ - cells ( $1 / 20$ versus $4 / 22)$. These results are in line with the view that a higher exo- to endocytosis ratio, particularly prominent in $h+$ cells, is the cause of the PM waviness.

Regarding PM curvature in fus $1 \Delta$ crosses, the contact region between cells was wider than in WT crosses with PMs of partner cells parallel over large surfaces (Fig. 6, A and B; and Fig. S5), consistent with a fusion delay. When the $h$ - was WT, it was the convex one in $92 \%(11 / 12)$ of asymmetric pairs and could protrude into the $h+$ fusl $\Delta$ cell $(6 / 12)$. When the $h$ - was fusl 1 , we recovered an equal portion of pairs with a convex $h$ - or $h+$ partner ( 6 and 6 ), though the asymmetry was overall poor. This suggests that the Fusl-dependent fusion focus is also important for the cell protrusive activity.

Previous measurements of fusion efficiencies in fusl $\Delta \times$ WT crosses suggested that the fusion defect is more pronounced when fusl is deleted in the $h+$ cell (Dudin et al., 2015). Indeed, while cell pair formation was not impaired upon fusl deletion in either cell type, fusion efficiency was significantly more compromised when the $h+$ cell was fusl $\Delta$ (Fig. $6 \mathrm{G}$ ). Because the main consequence of the loss of focalized secretion in fusl $\Delta$ is the inability to concentrate glucanases for $\mathrm{CW}$ digestion, this finding is in line with the idea that the $h+$ cells exhibit a stronger secretion activity. It also this raises the possibility that loss of the wPM more directly contributes to the enhanced fusion failure in $h-$ $\mathrm{WT} \times h+$ fusl $\Delta$ cell pairs.

\section{Cell protrusion requires strong turgor pressure}

A second hypothesis for the origin of PM asymmetries is that they may result from differences in turgor pressure in the two cell types. Specifically, higher turgor in the $h$ - cell may enhance PM tension and facilitate protrusion into the $h+$ cell.

To start testing this hypothesis, we made use of the gpdls mutant. Gpd1 is a glycerol-3-phosphate dehydrogenase necessary for glycerol production to restore turgor pressure in response to osmotic stress (Bernal et al., 2014; Ohmiya et al., 1995). Cells lacking gpdl exhibit reduced growth force when challenged in hyper-osmotic conditions (Minc et al., 2009). We repeated our ultrastructural studies in heterothallic crosses of WT expressing Fus1-sfGFP and mCherry-D4H to unlabeled gpd1 $\Delta$, generating 32 tomograms for $h-g p d l \Delta \times h+W T$ and 10 tomograms for $h-\mathrm{WT} \times h+$ gpdl $\Delta$ (Fig. 7, A and B; and Fig. S4 H). The frequency of PM waviness was not substantially altered in $h+$ gpdls prefusion cells $(63 \% ; 5 / 8)$ and increased in $h$ - cells $(55 \%$; 17/31; Fig. S4 I), and the waves were more prominent than in WT cells. LM confirmed that asymmetries in exo- and endocytosis were not altered in the gpdls cells: the exocytic Myo52 signal was higher and the endocytic Fiml signal lower in $h+$ cells, as in WT cells (Fig. 7, C and D). These data are consistent with a reduction in turgor pressure in gpdls cells exacerbating the PM waviness phenotype.

Regarding PM curvature, cell pairs in which the $h+$ cell was gpdls did not appear significantly different from WT. While our dataset is small, it showed the same tendencies as for WT pairs: both far CW contact cases were symmetric, and of the six close $\mathrm{CW}$ contact cases $(\mathrm{PM}$ distance $=47.5 \pm 30.7 \mathrm{~nm})$, two showed slight asymmetry, and three had the $h$ - cell protrude into a concave mutant $h+$ (Fig. S4 J). Thus, further decrease of turgor pressure in $h+$ does not alter, and may even accentuate, the asymmetry between prefusion partner cells.

By contrast, when the $h$ - cell was gpdl $\Delta$, most cell pairs showed a symmetric (16/31) or only slightly asymmetric (8/31) curvature configuration. The remaining pairs showed a clearer asymmetry (7/31), but we did not find any example of a cell protruding into its partner. Furthermore, among all asymmetric situations, both mating types had the same probability of adopting the convex shape (7 $h+$ versus $8 h-$; Fig. S4 J and Fig. 7 B). The vast majority of cell pairs showed substantial amount of CW between cells, with only five tomograms with minimal PM distance between cells $<100 \mathrm{~nm}$. However, the $h-$ gpdl $\times \mathrm{h}+$ WT pairs did not look like the WT far CW contact class, as the CWs of the two cells had merged together over a large surface area, leading to extended contact zones between cells. This suggests that the fusion process is delayed in these cell pairs. While fusion delay may reduce the chances of observing asymmetric configurations, we note that the pairs with cells closer than $100 \mathrm{~nm}$, including one with PM contact, were also symmetric or only very slightly asymmetric. These data suggest that reduction in turgor pressure in $h$ - cells prevents protrusion into the partner cell and leads to a more symmetric arrangement between partners.

To further investigate the importance of cell pair asymmetry on fusion success, we quantified mating and fusion efficiencies of gpdl $\Delta$ cells by LM. Deletion of gpdl in one or both mating types did not affect the ability of cells to pair together but caused opposite effects on fusion efficiency (Fig. 7, E and F): when the $h$ - cell lacked gpdl, which we showed above results in symmetric configurations, fusion was delayed; when the gpdl was deleted in the $h+$ cell, which preserves or accentuates asymmetry, fusion occurred faster than in WT pairs. Importantly, when both partner cells lacked gpdl, fusion efficiency was restored to WT levels, indicating that the changes in fusion efficiency observed upon deleting gpdl in only one partner cell reflect the difference in turgor pressure between the two cells rather than an absolute requirement of gpdl in either cell type. We conclude that asymmetries in turgor pressure between cell types promote cell fusion.

\section{Discussion}

By using correlative light-electron tomography, we reveal ultrastructural details of the fission yeast cell fusion process. While mating yeast cells are considered isogametes, which cannot be distinguished morphologically, our study now reveals that these cells display asymmetries at the ultrastructural level: 

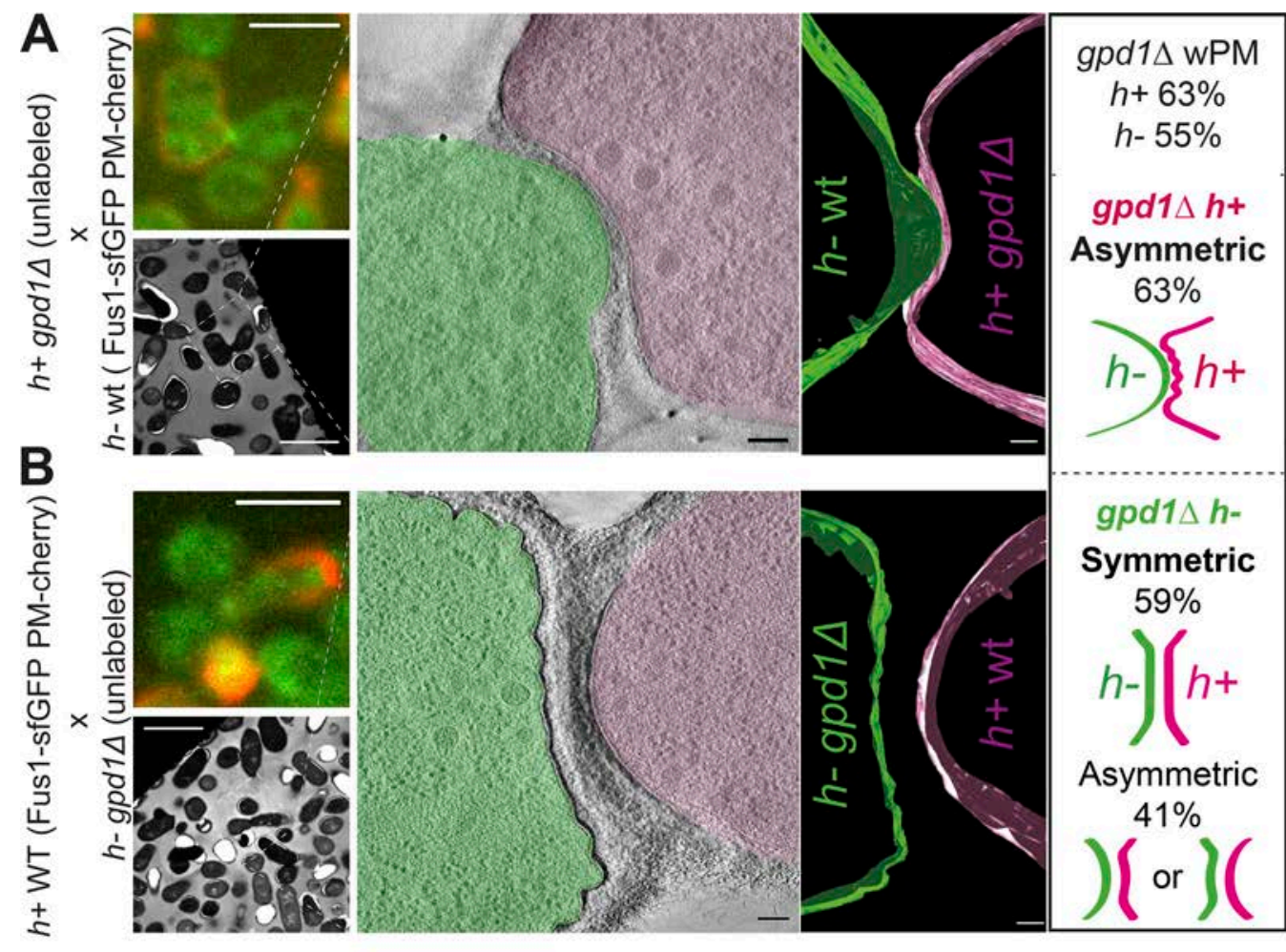

gpd1 14

Symmetric
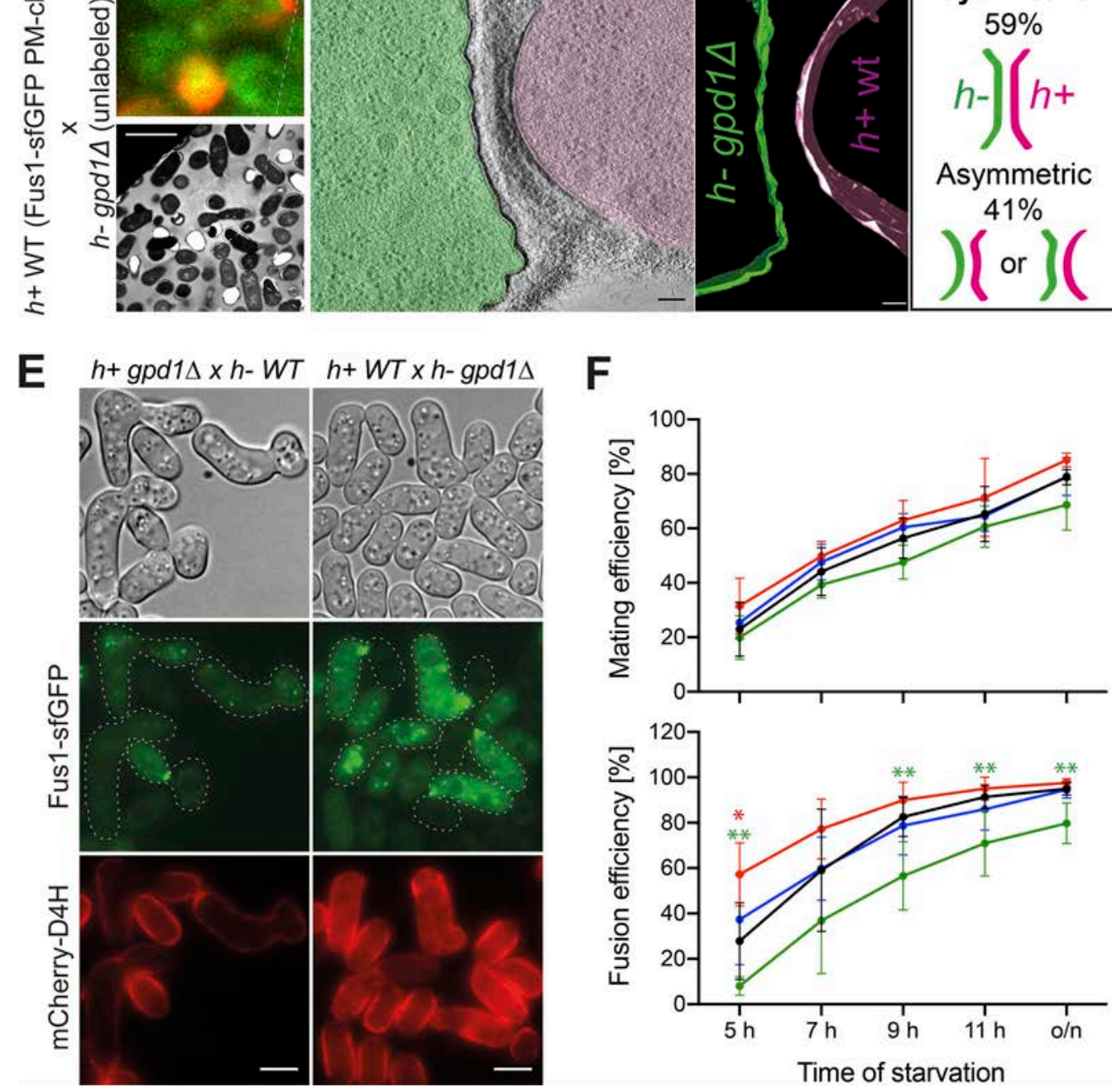

$\mathbf{F}$
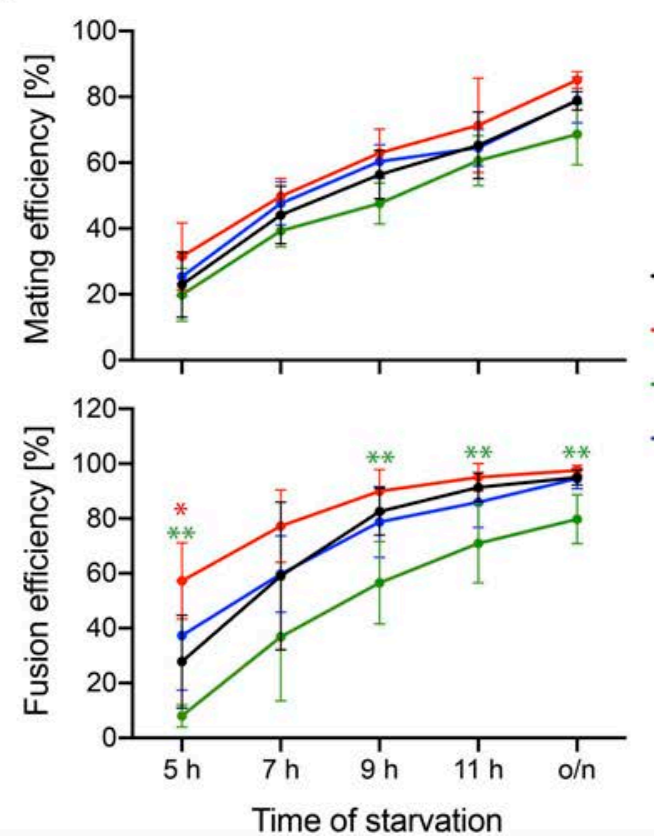

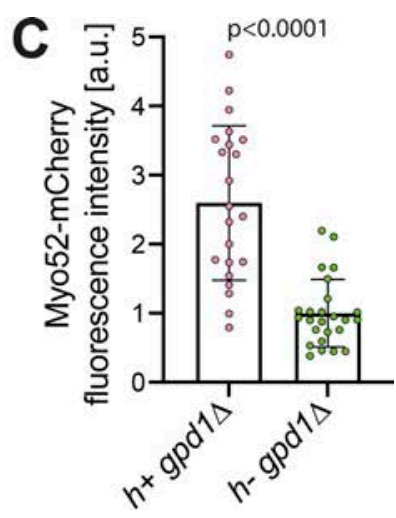

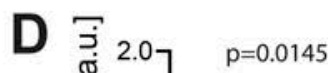

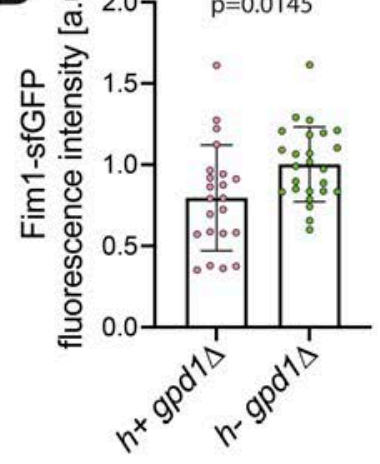

Figure 7. Different turgor pressure between cells of a pair is required for fusion. (A and B) Representative example of $h-W T$ cells crossed with $h+g p d 1 \Delta$ cells. The top left LM pictures show Fus1-sfGFP (green) and mCherry-D4H (red) expressed in the WT cell. The bottom left TEM pictures show the same mating pair. The virtual tomogram slices show PM shapes, segmented with Imod. Scale bars are $5 \mu \mathrm{m}$ in LM, $10 \mu \mathrm{m}$ in TEM, and $100 \mathrm{~nm}$ in tomogram slices. (C and D) Myo52-mCherry and Fim1-sfGFP mean fluorescence intensities of the 5 maximum pixels around each peak from shmoo tip profiles of tagged gpd1 $\Delta$ cells crossed to WT imaged as in Fig. 6, E and F ( $n=22 \mathrm{~h}+25 \mathrm{~h}$ - gpdl $\Delta$ cells from two experiments; P values are from Mann-Whitney tests). (E) LM images of WT $\times$ gpd1 $\Delta$ crosses at $7 \mathrm{~h}$ of nitrogen starvation. Fus1-sfGFP (green) and mCherry-D4H (red) are present only in WT cells. gpd1 $\Delta$ cells are unlabeled. (F) Mating and fusion efficiencies over time ( $n \geq 900$ cells of each genotype from five to seven experiments; ${ }^{*}, \mathrm{P}<0.05 ;{ }^{*}, \mathrm{P}<0.01$ from unpaired $t$ tests). 
one cell, generally the $h$ - partner, protrudes into the other cell, which usually exhibits a less tensed PM. Tomograms of cell pairs at or around the time of membrane merging further suggest how fusion pores form and expand during cell fusion of a walled species.

\section{Organization of the fusion focus}

The tomographic information reveals the fusion focus organization as a dense assembly of vesicles interspersed with linear filaments, largely excluding other organelles and ribosomes, in agreement with the localization of the formin Fusl, actinassociated proteins, myosin V Myo52, and other secretory vesicle-associated markers (Billault-Chaumartin and Martin, 2019; Dudin et al., 2015; Dudin et al., 2017). The filaments likely represent linear actin filaments, as the frequency of detection was reduced in cells lacking Fusl. We note that fusis cells contain other actin nucleators, so complete absence of filaments is not expected. There are two sub-populations of vesicles. The lower-abundance, smaller-size vesicles may represent endocytic vesicles, but we cannot exclude that they represent a mixed population with other transport carriers. The most abundant population, with diameters averaging $60-70 \mathrm{~nm}$, up to $100 \mathrm{~nm}$, represents secretory vesicles, as it is strongly diminished in cells lacking the formin Fusl, which concentrates the delivery of secretory markers at the cell growth projection (Dudin et al., 2015). Interestingly, the size of these vesicles is smaller than reported during mitotic growth, where the average diameter of secretory vesicles is $100 \mathrm{~nm}$ (Cheng et al., 2002; Wang et al., 2002; Wang et al., 2016). A similar observation was made in S. cerevisiae, where $60-70-\mathrm{nm}$ vesicles accumulate at mating projections and fusion sites (Baba et al., 1989; Gammie et al., 1998), but 100-nm vesicles are reported in mutants of the secretory pathway during vegetative growth (Novick and Schekman, 1979; Walworth and Novick, 1987). Thus, secretory vesicles have a reduced size, and perhaps specific contents including CW digestive enzymes, during sexual reproduction.

\section{Formation and expansion of the fusion pore}

Examination of fusion pores revealed three notable features. First, the majority of fusion pores captured in our tomograms were large, extending beyond the tomographic volume, with only three pores fully captured within this volume. Thus, early stages of membrane fusion and pore expansion are rapid relative to other steps. However, the existence of mutants that exhibit transient fusion indicates that this step is not absolutely unidirectional (Vještica et al., 2018). Second, the edges of fusion pores often did not appear circular. In larger pores, this manifested by the presence of a strand of CW penetrating into the pore. In tomograms of a pore edge along the section plane, these edges were often irregular with finger-like extensions into small regions lacking CW densities. We also identified such regions at cell contacts without an obvious pore, suggesting that these represent early stages of fusion, though the resolution limits due to resin embedding do not allow unambiguously tracing both PM leaflets around these features. Third, one tomogram displayed at least two such connections distant from each other, suggesting that membrane fusion can initiate at several positions. While merging of several expanding fusion pores may be the cause of the irregular edges, a second non-mutually exclusive possibility is that the rate of CW digestion limits fusion pore expansion (Fig. 3 F). As CW digestion depends on secreted enzymes (Dekker et al., 2004; Dudin et al., 2015; Dueñas-Santero et al., 2010; Martín-Cuadrado et al., 2003), secretion of individual vesicles may lead to nonhomogeneous enzyme release and cause degradation to occur at different rates around the pore edge.

\section{Control of PM tension during yeast mating}

A striking feature of prefusion cells is the wPM pattern. This pattern strongly correlates with local exocytosis. Indeed, PM waves are specifically observed at the site of cell-cell contact and are not seen in the lateral PM regions. The waves are shallower than $\Omega$-shapes formed immediately upon vesicle-PM fusion, but the observation that they are almost completely abolished in fusis cells argues that this pattern results from strong local secretion activity. In S. cerevisiae, undulating PM was also noted at the tips of mating projections (Baba et al., 1989), suggesting that this is a conserved feature during yeast sexual reproduction. The wavy pattern indicates reduced membrane tension, which does not instantaneously equilibrate with lateral membrane regions.

Interestingly, the occurrence of the wPM pattern increased through the fusion process and was more frequent in the $h+$ cell partner. This correlates with previous measurements showing increase in Myo52 levels in the course of fusion (Dudin et al., 2015) and with differences in the ratio of exo- to endocytic vesicles between cell types, where this ratio is higher in $h+$ than $h$ - cells, as measured on both EM and LM. Exo- and endocytosis are strongly correlated and mutually influence each other through changes in membrane tension, complicating the dissection of the cause of the cell type-specific difference. Indeed, studies in several cell types have shown that high membrane tension stimulates exocytosis, which in turn leads to tension reduction (Wang and Galli, 2018). Conversely, endocytosis is inhibited by membrane tension, contributing to its increase (Joseph and Liu, 2020). We envisage two possible scenarios. First, $h+$ cells may have a higher secretion rate. Consistent with this view, the number of exocytic vesicles and associated markers is higher in $h+$ cells, and this higher signal depends on Fusl, suggesting that the cell type-associated difference is inherent to the fusion focus. Previous data had also shown a faster turnover of Myo52 in fluorescence recovery after photobleaching experiments in $h+$ cells (Dudin et al., 2015). However, this scenario does not explain the lower amount of endocytic vesicles in $h+$ cells. A second scenario is that the cell type-specific difference resides in $h+$ cells having a lower maximal endocytic capacity. This may cause a membrane "traffic jam," where endocytosis cannot keep up with the exocytic capacity, leading to a local reduction of PM tension, manifested in PM waves, and a consequent decrease in the fusion of secretory vesicles with the PM, resulting in their accumulation. While further work will be required to distinguish between these possibilities, they both lead to the same overall conclusion that the balance between exo- and endocytosis is shifted toward higher exocytosis in $h+$ cells, which causes a local loss of PM tension at the cell front. 


\section{Control of cell protrusion by turgor pressure}

Our analysis revealed a strong asymmetry in membrane curvature at the site of cell contact, which increases as the CW thins, and culminates shortly before membrane fusion, with one cell, generally the $h$ - cell, protruding into its partner. The asymmetry is relaxed after fusion, as only few pairs with fusion pore showed asymmetry. Protruding $h$ - cells also showed an unresolved membrane invagination on either side of the protrusion. While the cause of this structural feature is unknown, its frequency suggests it may be important to help the organization of the fusion site.

We explored the hypothesis that higher turgor pressure in the protrusive cell may cause the protrusion. Indeed, deletion of Gpdl in $h$ - cells largely prevents their protrusion into $h+$ cells and yields more symmetrical fusion pairs. By contrast, its deletion in $h+$ cells does not prevent $h$ - cell protrusion. During vegetative growth, deletion of $\mathrm{Gpdl}$ reduces the growth force in hyper-osmotic conditions (Minc et al., 2009). During sexual reproduction, our results suggest that gpdl $\Delta$ cells have reduced growth force even without external hyper-osmotic challenge. This is consistent with Gpdl expression, which is induced upon osmotic shock (Aiba et al., 1995; Shiozaki and Russell, 1996; Wilkinson et al., 1996), but also increases about sixfold during mating (Mata and Bähler, 2006). Strong turgor is not the only requirement for protrusion, as disruption of the fusion focus in fusi $\Delta$ also interferes with formation of the protrusion in $h$ - cells. This suggests a role for the actin cytoskeleton in protrusion formation, which we hypothesize is indirect through delivery of digestive enzymes that weaken the CW. Thus, the fusion focus and higher turgor pressure in the $h$ - cell are both required for its asymmetric protrusion into $h+$ cells.

Regulation of turgor pressure may be generally important to control the fusion of walled cells. In S. cerevisiae, a mutual downregulation between the high osmolarity glycerol (HOG)-MAPK signaling and the pheromone-MAPK signaling has been described (Hall et al., 1996; Patterson et al., 2010; Vaga et al., 2014; Yamamoto et al., 2010). The pheromone-MAPK pathway also indirectly promotes HOG-MAPK signaling, by stimulating glycerol release through the glycerol efflux aquaglyceroporin Fpsl and consequent HOG-dependent glycerol synthesis to compensate for turgor loss (Baltanás et al., 2013). Interestingly, deletion of Fps1 in one, but not both, partner cell causes an increase in fusion intermediates, indicating a block or delay in fusion, though deletion of Gpd1 had no effect in this organism (Philips and Herskowitz, 1997). However, different from the situation in $S$. pombe, deletion of fpsl in either cell type leads to similar changes in cell contact site curvature, as observed by LM (Smith et al., 2017). Cytosolic protrusions of one cell into the other observed in the prml $\Delta$ mutant deficient in PM fusion are also reported to happen with equal frequency in either direction (Heiman and Walter, 2000). Thus, whereas our data indicate that $S$. pombe mating partners have different turgor pressures, the turgor pressure may be more equal between partner cells in S. cerevisiae.

\section{Origin of fission yeast gamete asymmetry}

An open question is how the asymmetry between cells comes about. Our analysis shows that ultrastructural asymmetry is linked with cell type: $h+$ cells have a higher exo- to endocytic ratio leading to low membrane tension, and $h$ - cells likely exhibit increased turgor pressure, which drives protrusion, likely also contributing to membrane tension increase. These cell typelinked differences are also visible at the functional level, as gpdls is detrimental only in $h$-cells. Thus, at least part of the reason for the asymmetry between the two cells is linked to their identity.

At the transcriptional level, there are very few differences between mating types. Only 16 genes are reportedly differentially expressed, most coding for agglutinins, pheromones, processing enzymes, and receptors (Mata and Bähler, 2006; Xue-Franzén et al., 2006). Cell type-specific differences may reside in the different pheromones and receptors: though acting through the same signaling pathway, they may elicit quantitatively different signals that alter crosstalk with the osmosensing pathway. Alternatively, turgor pressure differences may be linked to one uncharacterized small gene (SPAC1565.03), which is expressed specifically in $h+$ cells (Mata and Bähler, 2006; XueFranzén et al., 2006) and promotes growth on glycerol, like gpdl (Malecki et al., 2016). We note that gpdl expression itself, though induced during mating, is unlikely to be the key cell type-specific difference, as deletion of gpdl in both partner cells restores WTlike fusion kinetics and thus likely functional asymmetries.

Our analysis of heterothallic WT cells also revealed three cases of asymmetry reversal, where the $h+$ cell was protruding into the $h$ - partner, indicating that asymmetry is not completely hardwired. One possibility is that rapid fluctuations in the rates of exo/endocytosis around a higher average in the $h+$ cell underlie the presence of both wPM and SPM patterns in both cell types. Another possibility is that the cell type-imposed asymmetry is small and can be inverted by stochastic events. Because asymmetries increase during the course of fusion, small initial asymmetries may be amplified by cell interaction through either increase in signaling or mechanical signals upon contact with the partner cell.

\section{Function of membrane asymmetries}

Cell fusion is more strongly compromised by gpdl deletion in the $h$ - cell and fusl deletion in the $h+$ cell. These situations reduce the asymmetries observed in WT cells by preventing protrusion and PM waviness, respectively. This raises the question of a causal link between loss of asymmetry and fusion defects. Though PM waves may help initial membrane contact and merging, it is difficult to make a firm functional inference between membrane waves and fusion efficiency. Indeed, fus1 deletion or any other attempt to reduce local secretion to lessen PM waviness will also likely compromise the secretion of CW digestive enzymes. The functional importance of cell protrusion is easier to make, as gpdla causes fusion delays but only when solely deleted in the $h$ - cell. In addition, gpdl deletion only in the $h+$ cell, which maintains or exacerbates the protrusion asymmetry, leads to faster cell fusion. Thus, the different morphologies of the two cells are likely to promote efficient cell-cell fusion.

The asymmetry of yeast cell fusion recalls similar asymmetry observed in the fusion of Drosophila myoblasts, where fusioncompetent myoblasts form a podosome that protrudes into the 
myotube (Sens et al., 2010). Podosome-like structures are also observed in other types of somatic cell fusion (Brukman et al., 2019). Although the underlying protrusive force generation may be different (Martin, 2016), the similar configurations suggest that evolution of asymmetry is beneficial to the success of cell fusion. Future work should test whether local membrane curvature in wPM helps destabilize the bilayer and lower the energy required to initiate membrane merging. Cell protrusion promoted by turgor pressure and the fusion focus, which is initially countered by the intervening CW, may, upon complete CW removal, help overcome repulsive forces to bring the cells' PM close enough for merging. While the identity of the fusogenic machinery is still unknown, these ultrastructural PM features suggest that biophysical changes in membrane tension and curvature are important contributors to the membrane fusion process.

\section{Materials and methods}

\section{Strains and growth conditions}

S. pombe strains used in this study are listed in Table S1. Heterothallic $h+$ and $h$ - strains and homothallic ( $h 90)$ strains, able to switch mating types, were grown in minimum sporulation media (MSL) supplemented with nitrogen $(+\mathrm{N})$. Mating assays were conducted on MSL without nitrogen $(-\mathrm{N})$, essentially as described (Vjestica et al., 2016), except for gpd1 $\Delta$ strains, which were mated on malt extract (ME).

\section{Strain construction}

Strains were constructed using standard genetic manipulation of $S$. pombe either by tetrad dissection or transformation.

For generation of fus1 deletion mutants, WT strains were transformed with a linearized deletion plasmid (based on the pFA6a-kanMX backbone) containing at least $400 \mathrm{bp}$ of homology to fusl gene flanking regions (pSM1966). For generation of gpdl deletion mutants, the gpdl ORF was replaced by ura4 ORF. This was achieved by PCR amplification of a fragment from strain NM204 (received from Fred Chang, University of California, San Francisco, San Francisco, California) carrying ura4+ ORF at the gpdl locus, which was transformed and integrated in the genome of ura4-D18 strains by homologous recombination. For C-terminal tagging of myo52 with mCherry, strains were transformed with linearized fluorophore tagging plasmid containing at least $400 \mathrm{bp}$ of homology to the C-terminal part of the gene, in-frame mCherry, kanMX resistance, and myo52 3'UTR (pSM2735). For tagging fim1 with sfGFP, a pFA6a-sfGFP-natMX plasmid (pSM1686) was used as a template for PCR-based targeted tagging, as described (Bähler et al., 1998).

\section{Mating assays}

For both LM and CLEM experiments, cells were first precultured overnight in MSL $+\mathrm{N}$ at $25^{\circ} \mathrm{C}$, then diluted to $\mathrm{OD} 600=0.025$ into $\mathrm{MSL}+\mathrm{N}$ at $30^{\circ} \mathrm{C}$ for $16 \mathrm{~h}$. The amount of exponentially growing cells equivalent to OD600 of 3-5 (depending on the experiments but constant within the same experiment) was pelleted, washed in MSL- $\mathrm{N}$ by three rounds of centrifugation, and resuspended in 50-100 $\mu \mathrm{l}$ MSL-N. Cells were then placed in 10- $\mu$ l drops on $2 \%$ agar MSL-N or $2 \%$ agar $\mathrm{ME}$ plates at $30^{\circ} \mathrm{C}$ to allow mating.
Samples for CLEM were further processed after $5 \mathrm{~h}$ (see CLEM and tomography section below). Samples for time-lapse live imaging were transferred to MSL-N $2 \%$ agar pads after $4 \mathrm{~h}$ at $30^{\circ} \mathrm{C}$, mounted on a microscope slide, covered with a coverslip, sealed with VALAP (a mixture of vaseline, lanolin and paraffin; Vjestica et al., 2016), incubated one more hour on the pad at $30^{\circ} \mathrm{C}$, and imaged. Samples for experiments to measure mating and fusion efficiencies were placed on $\mathrm{ME}$ and maintained at $30^{\circ} \mathrm{C}$ for $5,7,9,11$, and $24 \mathrm{~h}$, and cells were imaged at the indicated times after mounting them on slides and covered with coverslips.

\section{LM}

LM images in Fig. $7 \mathrm{E}$ were obtained using wide-field microscopy performed on a DeltaVision platform (Cytiva) composed of a customized inverted microscope (IX-71; Olympus), a 100×/1.4 NA oil objective, a camera (CoolsNAP HQ2; Photometrics, or 4.2Mpx PrimeBSI sCMOS; Photometrics), and a color combined unit illuminator (Insight SSI 7; Social Science Insights). Images were acquired using softWoRx v4.1.2 software (Cytiva). Images shown are single-plane views.

Images presented in Fig. 5, C and D, or used to get data for Fig. 6, E and F; Fig. 7, C and D; and Fig. S4 D, are average projections of time-lapse videos acquired a maximum speed during 3 min using a spinning-disk microscope composed of an inverted microscope (DMI4000B; Leica) equipped with an HCX Plan Apochromat 100 $/ 1.46$ NA oil objective (PerkinElmer; including a real-time confocal scanning head [CSU22; Yokagawa Electric Corporation], solid-state laser lines, and an electron-multiplying charge coupled device camera [C9100; Hamamatsu Photonics]). Images were acquired using the Volocity software (PerkinElmer).

For CLEM, LM images for the WT h90 samples were acquired on a Nikon TE2000-E microscope equipped with a 100×/1.49 NA TIRF oil objective, a NEO sCMOS DC-152Q-C00-FI camera (Andor), and a Lambda DG-4 lamp (Sutter Instruments). Images were acquired using the NIS Elements software (Nikon). All other images were acquired on the DeltaVision platform described above, but with a UPlan Apochromat $60 \times / 1.42$ NA oil objective.

All LM images and time-lapse were acquired at room temperature $\left(\sim 22-23^{\circ} \mathrm{C}\right)$.

\section{CLEM and tomography}

CLEM was as described in Kukulski et al. (2012b), adapted for mating cells. Briefly, cells were grown for mating as described above. After washes to remove nitrogen, cells were added into MSL-N plates or ME plates for gpdl deletion mutant strains. We allowed cells to mate for $5 \mathrm{~h}$. A few microliters of MSL-N were pipetted onto the cells to form a thick slurry, which was pipetted onto a 3-mm-wide, 0.1-mm-deep specimen carrier (Wohlwend type A) closed with a flat lid (Wohlwend type B) for highpressure freezing with a HPM100 (Leica Microsystems; for h90 samples) or a Wohlwend HPF Compact 02. The carrier sandwich was disassembled in liquid nitrogen before freeze substitution. High-pressure frozen samples were processed by freeze substitution and embedding in Lowicryl HM2O using the Leica AFS 2 robot as described (Kukulski et al., 2012b). 300-nm sections were cut with a diamond knife using a Leica Ultracut $\mathrm{E}$ or Ultracut UC7 ultramicrotome, collected in $\mathrm{H}_{2} \mathrm{O}$, and picked 
up on carbon-coated 200-mesh copper grids (AGS160; Agar Scientific). For LM, the grid was inverted onto a 1× PBS drop on a microscope coverslip, which was mounted onto a microscope slide and imaged as indicated above. The grid was then recovered, rinsed in $\mathrm{H}_{2} \mathrm{O}$, and dried before post-staining with Reynolds lead citrate for $10 \mathrm{~min}$. 15-nm protein A-coupled gold beads were adsorbed to the top of the section as fiducials for tomography. TEMs were acquired on a FEI Tecnai 12 at $120 \mathrm{kV}$ using a bottom mount FEI Eagle camera (4kx4k). Low-magnification images were acquired at $17.816-\mathrm{nm}$ pixel size and high magnification at $1.205-\mathrm{nm}$ pixel size. A few tomography acquisitions (including panel 3D) were performed on a TF20 microscope (FEI) in scanning TEM mode, with an axial brightfield detector, using a camera length of $200 \mathrm{~mm}$ and a $50-\mu \mathrm{m} \mathrm{C2}$ aperture (Ader and Kukulski, 2017). For tomographic reconstruction of regions of interest, tilt series were acquired at $1.205-\mathrm{nm}$ pixel size (Tecnai) or 1.1-nm pixel size (TF20) over a tilt range as large as possible up to $\pm 60^{\circ}$ at $1^{\circ}$ increments using the Serial EM software (Mastronarde, 2005). The IMOD software package with gold fiducial alignment (Kremer et al., 1996; Mastronarde and Held, 2017) was used for tomogram reconstruction, segmentation, and modeling.

\section{Quantification and statistical analysis}

Minimal distance between PMs, and density and diameters of vesicles in tomograms were manually measured within a cylinder of diameter $1 \mu \mathrm{m}$ centered at the center of the cells' contact zone using IMOD software. Vesicle measurements were done for each cell separately in the half cylinder covering that cell.

Segmentation models of the PMs were done drawing the outer leaflets using IMOD software.

Waviness was assigned as follows: waves' amplitude (A) and wavelength $(\lambda)$ were measured using IMOD software. The presence of at least one wave with $\mathrm{A} \geq 5 \mathrm{~nm}$ and $2^{*} \mathrm{~A} / \lambda \geq 0.2$ was set as a threshold for the wPM pattern.

Myo52-tdTomato or mCherry, Fim1-sfGFP, and Exo84-GFP fluorescence profiles at shmoo tips were obtained on average projections of time-lapse videos acquired at maximum speed during $3 \mathrm{~min}$. Fluorescence intensity along a 7-pixel-wide segmented line was collected using the FIJI plot profile tool. Values were corrected for the external background. The curves were centered on the maximum pixel values of the Myo52 channel. Values in Fig. 5 F; Fig. 6 E, right; Fig. 6 F, right; Fig. 7, E and F; and Fig. S5 F were obtained from the average of the 5 pixels around each peak maximum from the profiles described above.

Mating and fusion efficiencies were calculated as in Dudin et al. (2015): mating efficiency represents the fraction of cells engaged in mating; fusion efficiency represents the fraction of mating pairs that have fused. Mating pairs were identified by the presence of Fusl-sfGFP or Myo52-tdTomato at the fusion focus. The transfer of mCherry-D4H or Fimbrin1-sfGFP into the unlabeled partner was used to identify fused pairs.

Statistical analysis was performed using GraphPad Prism. Statistical significance was determined using one-way ANOVA test, paired or unpaired $t$ test, or Mann-Whitney test, as indicated. In all cases, Gaussian data distribution was tested using the D'Agostino-Pearson normality test.

\section{Online supplemental material}

Fig. S1 shows examples of CLEM and ultrastructural features during mating. Fig. S2 shows the shape of the fusion pore. Fig. S3 shows PM asymmetries during cell fusion. Fig. S4 shows classification of tomograms from heterothallic crosses and additional data on distribution of exocytic vesicles. Fig. S5 shows morphology of WT $\times$ fusi $\Delta$ crosses. Video 1 shows a tomogram and model of a cell pair prefusion. Video 2 shows a tomogram and model of a cell pair after fusion. Video 3 shows a tomogram and model of a cell pair with several connections. Video 4 shows a tomogram and model of a cell pair with an asymmetric pore. Video 5 shows a tomogram and model of a cell pair with an asymmetric pore. Video 6 shows a tomogram and model of a cell pair with a pore containing a strand of CW. Table S1 lists yeast strains used in this study.

\section{Acknowledgments}

We thank the University of Lausanne EM facility, in particular Jean Daraspe, for help with EM sample preparation and acquisition, Bruno Humbel for help with manipulation of the electron microscope, and Christel Genoud for discussions and suggestions. We thank the EM and LM facilities of the Medical Research Council Laboratory of Molecular Biology for microscopy support. We thank Fred Chang (University of California, San Francisco, San Francisco, California) for sharing a gpdlı strain and Alejandro Melero and Laura Merlini for comments on the manuscript.

This work was funded by a European Research Council Consolidator grant (CellFusion) and a Swiss National Science Foundation grant (310030B_176396) to S.G. Martin, and a "poste de soutien" awarded to O. Muriel. W. Kukulski was supported by the Medical Research Council (MC_UP_1201/8). S.G. Martin also thanks the Herbette Foundation (University of Lausanne) for support of her sabbatical visit in the lab of W. Kukulski.

The authors declare no competing financial interests.

Author contributions: S.G. Martin prepared CLEM samples and reconstructed tomograms for the $h 90$ cells with help from W. Kukulski. O. Muriel acquired EM data for all samples except for a few acquired by W. Kukulski, and prepared CLEM samples and reconstructed tomograms for all heterothallic crosses. 0 . Muriel performed all other experiments with technical support from L. Michon. O. Muriel analyzed the data together with S.G. Martin. O. Muriel and S.G. Martin prepared figures and wrote the manuscript, with comments from W. Kukulski. O. Muriel and S.G. Martin acquired funding.

Submitted: 22 March 2021

Revised: 15 July 2021

Accepted: 26 July 2021

\section{References}

Ader, N.R., and W. Kukulski. 2017. triCLEM: Combining high-precision, room temperature CLEM with cryo-fluorescence microscopy to identify very rare events. Methods Cell Biol. 140:303-320. https://doi.org/10.1016/bs .mcb.2017.03.009

Aiba, H., H. Yamada, R. Ohmiya, and T. Mizuno. 1995. The osmo-inducible gpdl+ gene is a target of the signaling pathway involving Wisl MAP- 
kinase kinase in fission yeast. FEBS Lett. 376:199-201. https://doi.org/10 .1016/0014-5793(95)01277-4

Atilgan, E., V. Magidson, A. Khodjakov, and F. Chang. 2015. Morphogenesis of the Fission Yeast Cell through Cell Wall Expansion. Curr. Biol. 25: 2150-2157. https://doi.org/10.1016/j.cub.2015.06.059

Baba, M., N. Baba, Y. Ohsumi, K. Kanaya, and M. Osumi. 1989. Threedimensional analysis of morphogenesis induced by mating pheromone alpha factor in Saccharomyces cerevisiae. J. Cell Sci. 94:207-216. https://doi.org/10.1242/jcs.94.2.207

Bähler, J., J.Q. Wu, M.S. Longtine, N.G. Shah, A. McKenzie III, A.B. Steever, A. Wach, P. Philippsen, and J.R. Pringle. 1998. Heterologous modules for efficient and versatile PCR-based gene targeting in Schizosaccharomyces pombe. Yeast. 14:943-951. https://doi.org/10.1002/(SICI)1097 -0061(199807)14:10<943::AID-YEA292>3.0.CO;2-Y

Baltanás, R., A. Bush, A. Couto, L. Durrieu, S. Hohmann, and A. ColmanLerner. 2013. Pheromone-induced morphogenesis improves osmoadaptation capacity by activating the HOG MAPK pathway. Sci. Signal. 6: ra26. https://doi.org/10.1126/scisignal.2003312

Bernal, M., J. Zhurinsky, A.B. Iglesias-Romero, M.A. Sanchez-Romero, I. FlorParra, L. Tomas-Gallardo, A.J. Perez-Pulido, J. Jimenez, and R.R. Daga. 2014. Proteome-wide search for PP2A substrates in fission yeast. Proteomics. 14:1367-1380. https://doi.org/10.1002/pmic.201300136

Billault-Chaumartin, I., and S.G. Martin. 2019. Capping Protein Insulates Arp2/3-Assembled Actin Patches from Formins. Curr. Biol. 29:3165-3176.e6. https://doi.org/10.1016/j.cub.2019.07.088

Brukman, N.G., B. Uygur, B. Podbilewicz, and L.V. Chernomordik. 2019. How cells fuse. J. Cell Biol. 218:1436-1451. https://doi.org/10.1083/ jcb.201901017

Chan, K.M.C., S. Son, E.M. Schmid, and D.A. Fletcher. 2020. A viral fusogen hijacks the actin cytoskeleton to drive cell-cell fusion. eLife. 9:e51358. https://doi.org/10.7554/eLife.51358

Cheng, H., R. Sugiura, W. Wu, M. Fujita, Y. Lu, S.O. Sio, R. Kawai, K. Takegawa, H. Shuntoh, and T. Kuno. 2002. Role of the Rab GTP-binding protein Ypt3 in the fission yeast exocytic pathway and its connection to calcineurin function. Mol. Biol. Cell. 13:2963-2976. https://doi.org/10 $.1091 / \mathrm{mbc} .01-09-0463$

Dekker, N., D. Speijer, C.H. Grün, M. van den Berg, A. de Haan, and F. Hochstenbach. 2004. Role of the alpha-glucanase Agnlp in fission-yeast cell separation. Mol. Biol. Cell. 15:3903-3914. https://doi.org/10.1091/ mbc.e04-04-0319

Dudin, O., F.O. Bendezú, R. Groux, T. Laroche, A. Seitz, and S.G. Martin. 2015. A formin-nucleated actin aster concentrates cell wall hydrolases for cell fusion in fission yeast. J. Cell Biol. 208:897-911. https://doi.org/10.1083/ jcb.201411124

Dudin, O., L. Merlini, and S.G. Martin. 2016. Spatial focalization of pheromone/MAPK signaling triggers commitment to cell-cell fusion. Genes Dev. 30:2226-2239. https://doi.org/10.1101/gad.286922.116

Dudin, O., L. Merlini, F.O. Bendezú, R. Groux, V. Vincenzetti, and S.G. Martin. 2017. A systematic screen for morphological abnormalities during fission yeast sexual reproduction identifies a mechanism of actin aster formation for cell fusion. PLoS Genet. 13:e1006721. https://doi.org/10 .1371/journal.pgen.1006721

Dueñas-Santero, E., A.B. Martín-Cuadrado, T. Fontaine, J.P. Latgé, F. del Rey, and C. Vázquez de Aldana. 2010. Characterization of glycoside hydrolase family 5 proteins in Schizosaccharomyces pombe. Eukaryot. Cell. 9: 1650-1660. https://doi.org/10.1128/EC.00187-10

Gammie, A.E., V. Brizzio, and M.D. Rose. 1998. Distinct morphological phenotypes of cell fusion mutants. Mol. Biol. Cell. 9:1395-1410. https://doi .org/10.1091/mbc.9.6.1395

Goodenough, U.W., P.A. Detmers, and C. Hwang. 1982. Activation for cell fusion in Chlamydomonas: analysis of wild-type gametes and nonfusing mutants. J. Cell Biol. 92:378-386. https://doi.org/10.1083/jcb.92.2.378

Hall, J.P., V. Cherkasova, E. Elion, M.C. Gustin, and E. Winter. 1996. The osmoregulatory pathway represses mating pathway activity in Saccharomyces cerevisiae: isolation of a FUS3 mutant that is insensitive to the repression mechanism. Mol. Cell. Biol. 16:6715-6723. https://doi.org/ 10.1128/MCB.16.12.6715

Heiman, M.G., and P. Walter. 2000. Prmlp, a pheromone-regulated multispanning membrane protein, facilitates plasma membrane fusion during yeast mating. J. Cell Biol. 151:719-730. https://doi.org/10.1083/jcb.151.3.719

Hernández, J.M., and B. Podbilewicz. 2017. The hallmarks of cell-cell fusion. Development. 144:4481-4495. https://doi.org/10.1242/dev.155523

Joseph, J.G., and A.P. Liu. 2020. Mechanical Regulation of Endocytosis: New Insights and Recent Advances. Adv. Biosyst. 4:e1900278. https://doi.org/ 10.1002/adbi.201900278
Kim, J.H., and E.H. Chen. 2019. The fusogenic synapse at a glance. J. Cell Sci. 132:jcs213124. https://doi.org/10.1242/jcs.213124

Kim, J.H., Y. Ren, W.P. Ng, S. Li, S. Son, Y.S. Kee, S. Zhang, G. Zhang, D.A. Fletcher, D.N. Robinson, and E.H. Chen. 2015. Mechanical tension drives cell membrane fusion. Dev. Cell. 32:561-573. https://doi.org/10 .1016/j.devcel.2015.01.005

Kremer, J.R., D.N. Mastronarde, and J.R. McIntosh. 1996. Computer visualization of three-dimensional image data using IMOD. J. Struct. Biol. 116: 71-76. https://doi.org/10.1006/jsbi.1996.0013

Kukulski, W., M. Schorb, M. Kaksonen, and J.A. Briggs. 2012a. Plasma membrane reshaping during endocytosis is revealed by time-resolved electron tomography. Cell. 150:508-520. https://doi.org/10.1016/j.cell .2012 .05 .046

Kukulski, W., M. Schorb, S. Welsch, A. Picco, M. Kaksonen, and J.A. Briggs. 2012b. Precise, correlated fluorescence microscopy and electron tomography of lowicryl sections using fluorescent fiducial markers. Methods Cell Biol. 111:235-257. https://doi.org/10.1016/B978-0-12-416026 $-2.00013-3$

Malecki, M., D.A. Bitton, M. Rodríguez-López, C. Rallis, N.G. Calavia, G.C. Smith, and J. Bähler. 2016. Functional and regulatory profiling of energy metabolism in fission yeast. Genome Biol. 17:240. https://doi.org/10 $.1186 /$ s13059-016-1101-2

Marek, M., V. Vincenzetti, and S.G. Martin. 2020. Sterol biosensor reveals LAM-family Ltcl-dependent sterol flow to endosomes upon Arp $2 / 3$ inhibition. J. Cell Biol. 219:e202001147. https://doi.org/10.1083/jcb .202001147

Martin, S.G. 2016. Role and organization of the actin cytoskeleton during cellcell fusion. Semin. Cell Dev. Biol. 60:121-126. https://doi.org/10.1016/j semcdb.2016.07.025

Martín-Cuadrado, A.B., E. Dueñas, M. Sipiczki, C.R. Vázquez de Aldana, and F. del Rey. 2003. The endo-beta-1,3-glucanase englp is required for dissolution of the primary septum during cell separation in Schizosaccharomyces pombe. J. Cell Sci. 116:1689-1698. https://doi.org/10 $.1242 /$ jcs.00377

Mastronarde, D.N. 2005. Automated electron microscope tomography using robust prediction of specimen movements. J. Struct. Biol. 152:36-51. https://doi.org/10.1016/j.jsb.2005.07.007

Mastronarde, D.N., and S.R. Held. 2017. Automated tilt series alignment and tomographic reconstruction in IMOD. J. Struct. Biol. 197:102-113. https:// doi.org/10.1016/j.jsb.2016.07.011

Mata, J., and J. Bähler. 2006. Global roles of Stellp, cell type, and pheromone in the control of gene expression during early sexual differentiation in fission yeast. Proc. Natl. Acad. Sci. USA. 103:15517-15522. https://doi.org/ 10.1073/pnas.0603403103

Merlini, L., O. Dudin, and S.G. Martin. 2013. Mate and fuse: how yeast cells do it. Open Biol. 3:130008. https://doi.org/10.1098/rsob.130008

Merlini, L., B. Khalili, O. Dudin, L. Michon, V. Vincenzetti, and S.G. Martin 2018. Inhibition of Ras activity coordinates cell fusion with cell-cell contact during yeast mating. J. Cell Biol. 217:1467-1483. https://doi.org/ $10.1083 /$ jcb.201708195

Minc, N., A. Boudaoud, and F. Chang. 2009. Mechanical forces of fission yeast growth. Curr. Biol. 19:1096-1101. https://doi.org/10.1016/j.cub.2009.05.031

Novick, P., and R. Schekman. 1979. Secretion and cell-surface growth are blocked in a temperature-sensitive mutant of Saccharomyces cerevisiae. Proc. Natl. Acad. Sci. USA. 76:1858-1862. https://doi.org/10.1073/ pnas.76.4.1858

Ohmiya, R., H. Yamada, K. Nakashima, H. Aiba, and T. Mizuno. 1995. Osmoregulation of fission yeast: cloning of two distinct genes encoding glycerol-3-phosphate dehydrogenase, one of which is responsible for osmotolerance for growth. Mol. Microbiol. 18:963-973. https://doi.org/10 $.1111 / \mathrm{j} .1365-2958.1995 .18050963 . \mathrm{x}$

Patterson, J.C., E.S. Klimenko, and J. Thorner. 2010. Single-cell analysis reveals that insulation maintains signaling specificity between two yeast MAPK pathways with common components. Sci. Signal. 3:ra75. https:// doi.org/10.1126/scisignal.2001275

Philips, J., and I. Herskowitz. 1997. Osmotic balance regulates cell fusion during mating in Saccharomyces cerevisiae. J. Cell Biol. 138:961-974. https://doi.org/10.1083/jcb.138.5.961

Seike, T., H. Maekawa, T. Nakamura, and C. Shimoda. 2019a. The asymmetric chemical structures of two mating pheromones reflect their differential roles in mating of fission yeast. J. Cell Sci. 132:jcs.230722. https://doi .org/10.1242/jcs.230722

Seike, T., C. Shimoda, and H. Niki. 2019b. Asymmetric diversification of mating pheromones in fission yeast. PLoS Biol. 17:e3000101. https://doi .org/10.1371/journal.pbio.3000101 
Sens, K.L., S. Zhang, P. Jin, R. Duan, G. Zhang, F. Luo, L. Parachini, and E.H. Chen. 2010. An invasive podosome-like structure promotes fusion pore formation during myoblast fusion. J. Cell Biol. 191:1013-1027. https://doi .org/10.1083/jcb.201006006

Shilagardi, K., S. Li, F. Luo, F. Marikar, R. Duan, P. Jin, J.H. Kim, K. Murnen, and E.H. Chen. 2013. Actin-propelled invasive membrane protrusions promote fusogenic protein engagement during cell-cell fusion. Science. 340:359-363. https://doi.org/10.1126/science.1234781

Shiozaki, K., and P. Russell. 1996. Conjugation, meiosis, and the osmotic stress response are regulated by Spcl kinase through Atfl transcription factor in fission yeast. Genes Dev. 10:2276-2288. https://doi.org/10.1101/gad.10 .18 .2276

Smith, J.A., A.E. Hall, and M.D. Rose. 2017. Membrane curvature directs the localization of Cdc42p to novel foci required for cell-cell fusion. J. Cell Biol. 216:3971-3980. https://doi.org/10.1083/jcb.201703169

Vaga, S., M. Bernardo-Faura, T. Cokelaer, A. Maiolica, C.A. Barnes, L.C. Gillet, B. Hegemann, F. van Drogen, H. Sharifian, E. Klipp, et al. 2014. Phosphoproteomic analyses reveal novel cross-modulation mechanisms between two signaling pathways in yeast. Mol. Syst. Biol. 10:767. https:// doi.org/10.15252/msb.20145112

Vjestica, A., L. Merlini, O. Dudin, F.O. Bendezu, and S.G. Martin. 2016. Microscopy of Fission Yeast Sexual Lifecycle. J. Vis. Exp. (109):53801. https://doi.org/10.3791/53801

Vještica, A., L. Merlini, P.J. Nkosi, and S.G. Martin. 2018. Gamete fusion triggers bipartite transcription factor assembly to block re-fertilization. Nature. 560:397-400. https://doi.org/10.1038/s41586-018-0407-5
Walworth, N.C., and P.J. Novick. 1987. Purification and characterization of constitutive secretory vesicles from yeast. J. Cell Biol. 105:163-174. https://doi.org/10.1083/jcb.105.1.163

Wang, G., and T. Galli. 2018. Reciprocal link between cell biomechanics and exocytosis. Traffic. 19:741-749. https://doi.org/10.1111/tra.12584

Wang, H., X. Tang, J. Liu, S. Trautmann, D. Balasundaram, D. McCollum, and M.K. Balasubramanian. 2002. The multiprotein exocyst complex is essential for cell separation in Schizosaccharomyces pombe. Mol. Biol. Cell. 13:515-529. https://doi.org/10.1091/mbc.01-11-0542

Wang, N., I.J. Lee, G. Rask, and J.Q. Wu. 2016. Roles of the TRAPP-II Complex and the Exocyst in Membrane Deposition during Fission Yeast Cytokinesis. PLOS Biol. 14:e1002437. https://doi.org/10.1371/journal.pbio .1002437

Wilkinson, M.G., M. Samuels, T. Takeda, W.M. Toone, J.C. Shieh, T. Toda, J.B. Millar, and N. Jones. 1996. The Atfl transcription factor is a target for the Styl stress-activated MAP kinase pathway in fission yeast. Genes Dev. 10:2289-2301. https://doi.org/10.1101/gad.10.18.2289

Xue-Franzén, Y., S. Kjaerulff, C. Holmberg, A. Wright, and O. Nielsen. 2006. Genomewide identification of pheromone-targeted transcription in fission yeast. BMC Genomics. 7:303. https://doi.org/10.1186/1471-2164 $-7-303$

Yamamoto, K., K. Tatebayashi, K. Tanaka, and H. Saito. 2010. Dynamic control of yeast MAP kinase network by induced association and dissociation between the Ste50 scaffold and the Opy2 membrane anchor. Mol. Cell. 40:87-98. https://doi.org/10.1016/j.molcel.2010 .09 .011 


\section{Supplemental material}


A

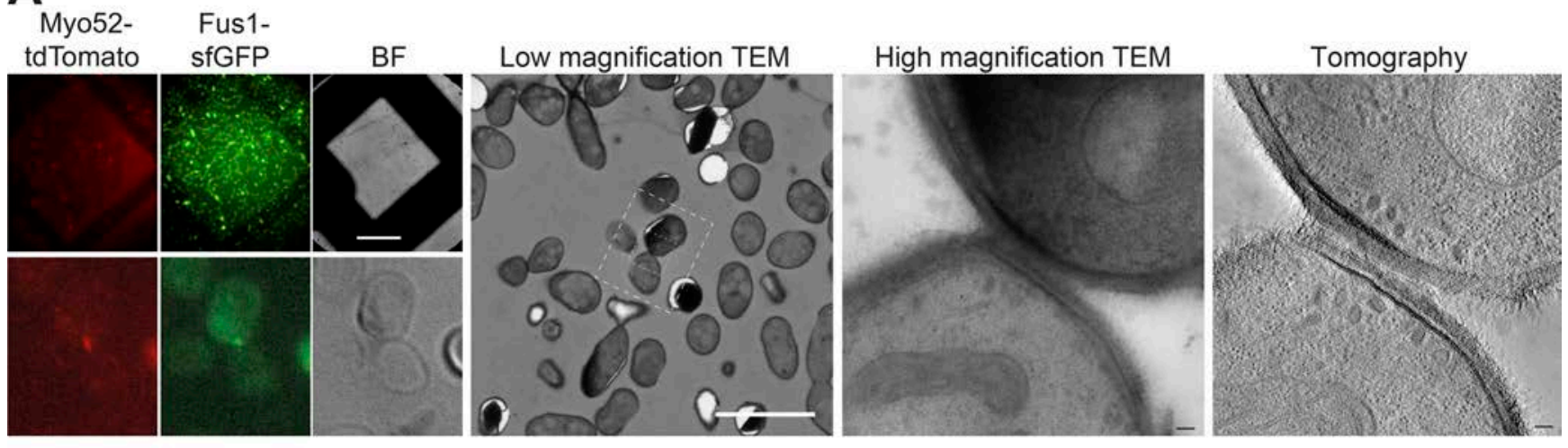

B
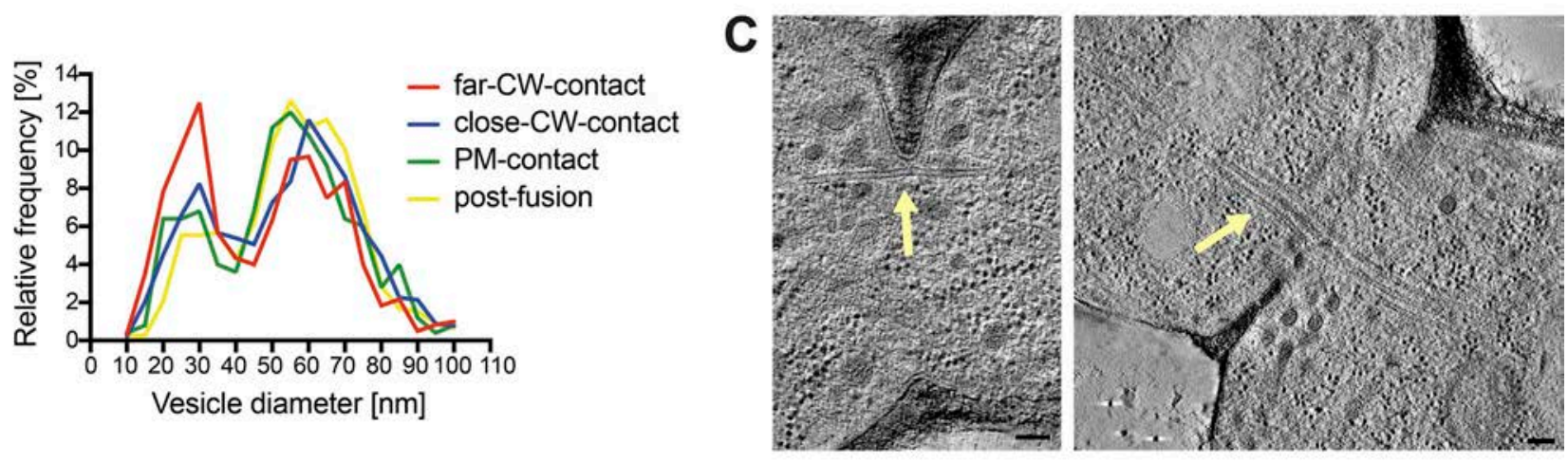

D

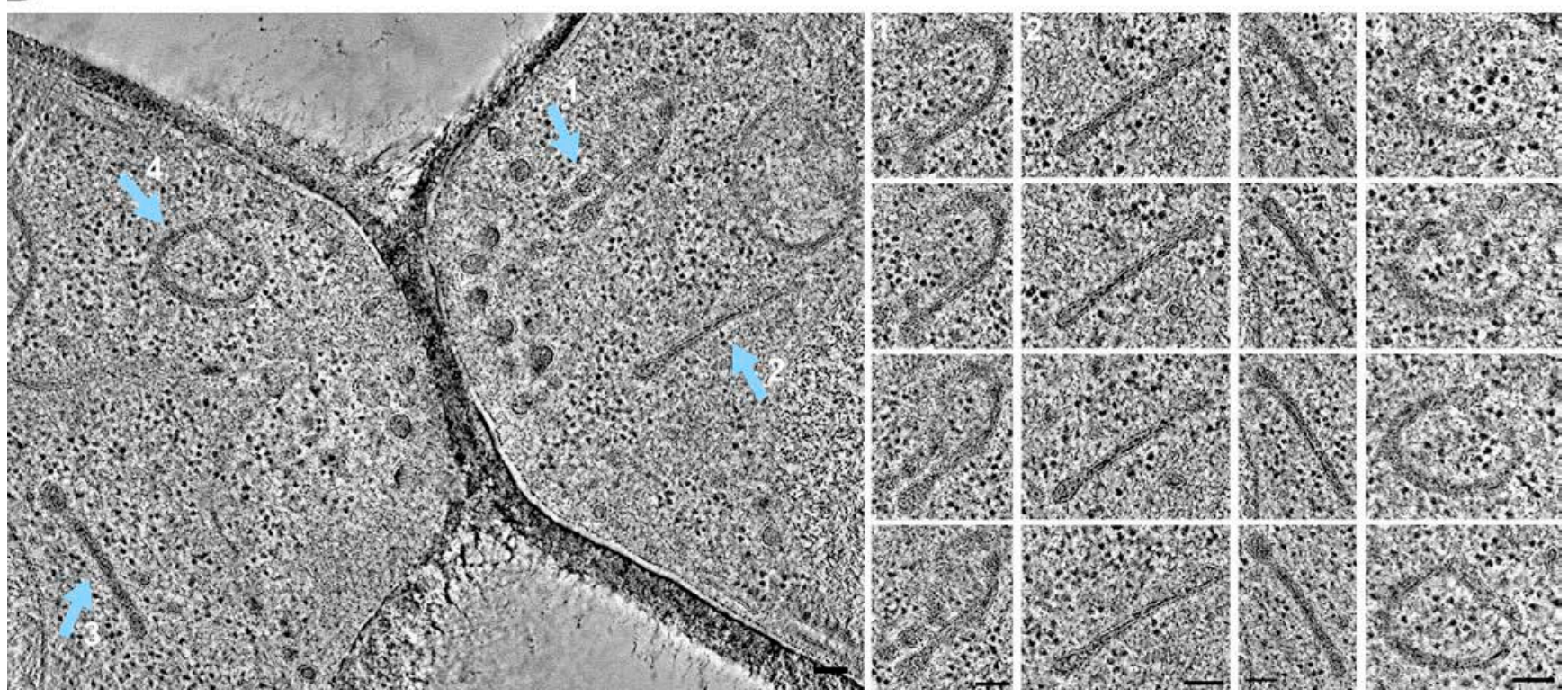

Figure S1. Examples of CLEM and ultrastructural features during mating. (A) LM images are GFP and tdTomato signals on 300-nm sections of resinembedded yeast cells expressing Fus1-GFP and Myo52-tdTomato, placed on EM grids. Scale bar is $45 \mu \mathrm{m}$. Cells with fusion focus fluorescence signal are identified in low magnification (17.816-nm pixel size) TEM according to their position, preservation is tested at high magnification (1.205-nm pixel size) TEM, and tilt series are acquired at this same magnification and processed for tomographic 3D reconstruction. Scale bars are $10 \mu \mathrm{m}$ in low magnification TEM and $100 \mathrm{~nm}$ in high magnification TEM and virtual z-slice through tomogram. BF, brightfield. (B) Size distribution of vesicles separated in the four stages: far CW contact ( $n=20$ cells; 616 vesicles), CW contact close ( $n=60$ cells; 1,865 vesicles), PM contact ( $n=10$ cells; 252 vesicles), and after fusion ( $n=16 ; 788$ vesicles). Bin width $=5 \mathrm{~nm}$. (C and D) Virtual z-slices through electron tomograms of mating cells showing microtubule bundles crossing the fusion pore (C, yellow arrow) and organelles with similar density to secretory vesicles, organized in sheets or reticulated structures (D, blue arrows). Scale bars for EM images, $100 \mathrm{~nm}$. 

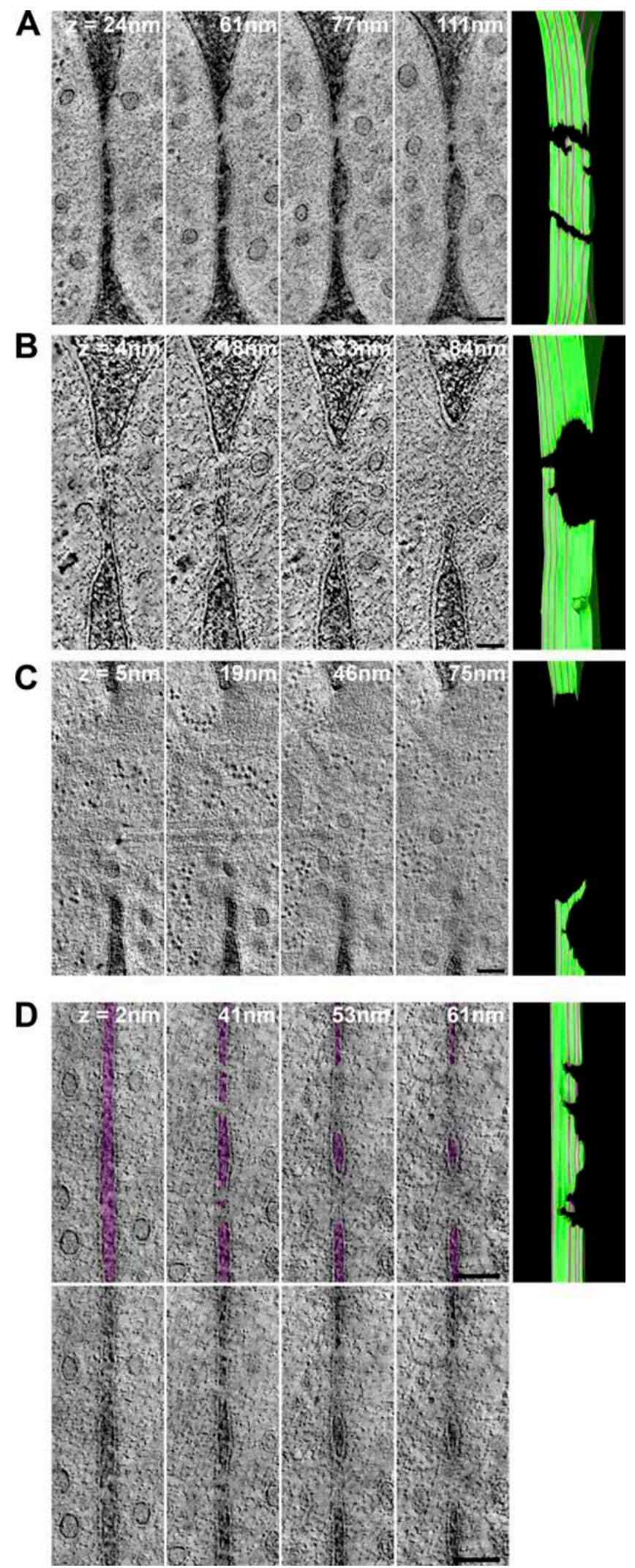

Figure S2. Shape of the fusion pore. (A-C) Original noncolored virtual z-slices through tomograms shown in Fig. 3, C and D. (D) Four virtual z-slices through a tomogram showing two large pores with irregular edges. These two pores are likely joined in a single pore outside the reconstructed tomographic volume. On the top line, the CW is colored in transparent purple to help visualization. Corresponding models of the outer leaflet of the PM are on the right. The position of each z-slice is indicated by the purple lines on the model, where the z-slice intersects the green PM outline at perpendicular angle. Z-slices are ordered from bottom (left edge of the model image) to top (right edge; see Video 3, Video 4, Video 5, and Video 6). Scale bars, $100 \mathrm{~nm}$. 
A

\begin{tabular}{|c|c|c|c|c|c|c|}
\hline h90 & $\mathrm{N}$ tomograms & PM layout & wPM both & sPM both & wPM vs sPM & Total \\
\hline \multirow{3}{*}{ far-CW-contact } & \multirow{3}{*}{13} & Convex vs. convex / flat vs. flat & 0 & 9 & 2 & 11 \\
\hline & & Convex vs. flat & 0 & 1 & 1 & 2 \\
\hline & & Convex vs. concave & 0 & 0 & 0 & 0 \\
\hline \multirow{3}{*}{ close-CW-contact } & \multirow{3}{*}{50} & Convex vs. convex / flat vs. flat & 4 & 10 & 13 & 27 \\
\hline & & Convex vs. flat & 0 & 1 & 9 & 10 \\
\hline & & Convex vs. concave & 0 & 3 & 10 & 13 \\
\hline \multirow{3}{*}{ PM-contact } & \multirow{3}{*}{11} & Convex vs. convex / flat vs. flat & 0 & 1 & 1 & 2 \\
\hline & & Convex vs. flat & 0 & 0 & 2 & 2 \\
\hline & & Convex vs. concave & 0 & 1 & 6 & 7 \\
\hline \multirow{3}{*}{ Post-fusion } & \multirow{3}{*}{43} & Convex vs. convex / flat vs. flat & 1 & 32 & 4 & 37 \\
\hline & & Convex vs. flat & 0 & 0 & 0 & 0 \\
\hline & & Convex vs. concave & 2 & 4 & 0 & 6 \\
\hline
\end{tabular}

B

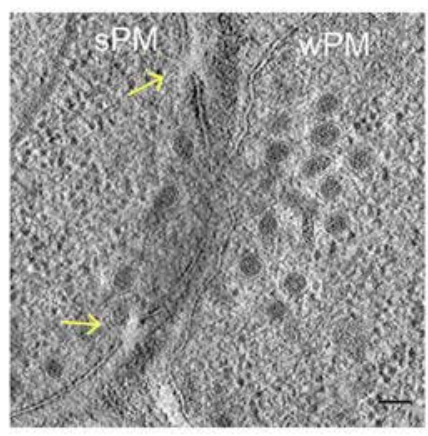

close-CW-contact
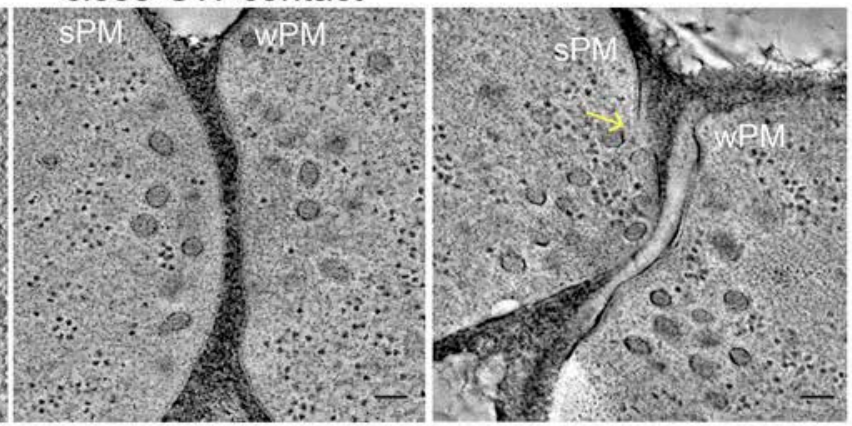

C

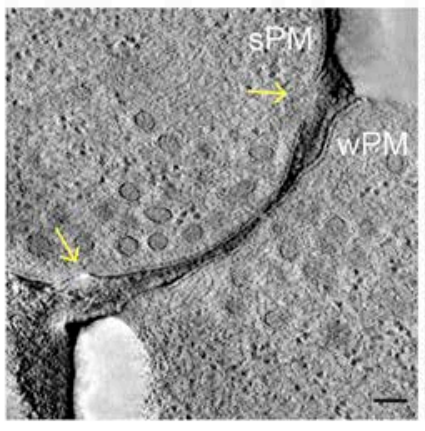

PM-contact
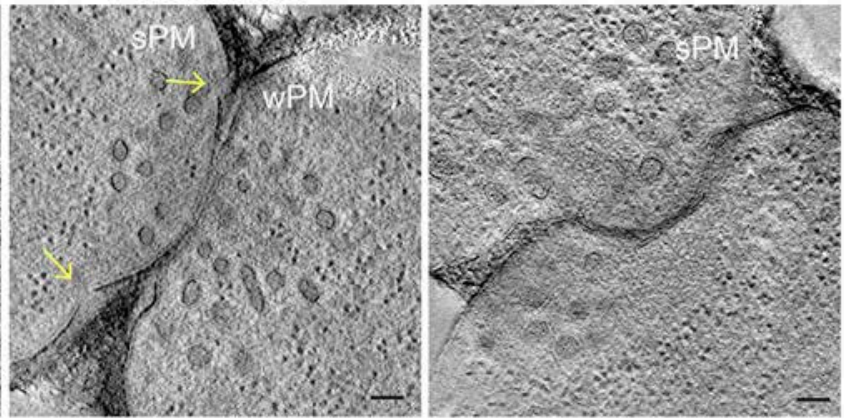

D

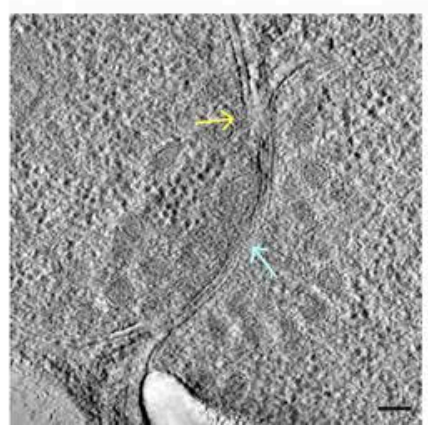

Post-fusion
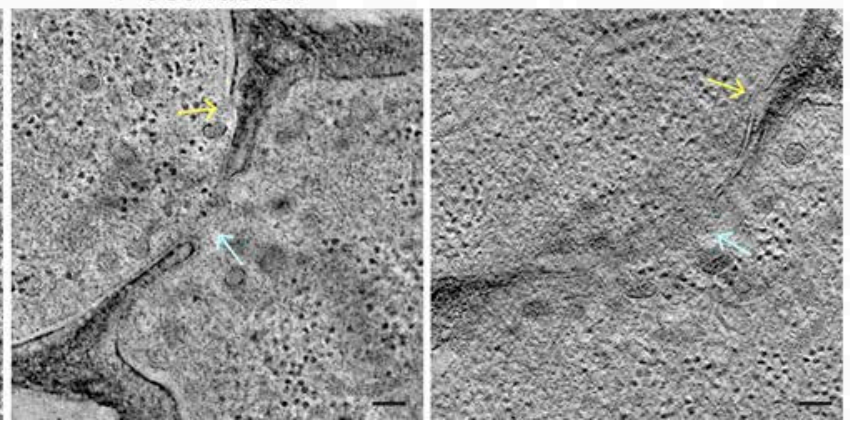

Figure S3. PM asymmetries during cell fusion. (A) Table classifying the set of tomograms from h90 WT cells used in this study, according to PM morphology along the four stages of fusion. (B-D) Virtual z-slices through tomograms of mating cells showing PM asymmetries (convex versus concave) in close CW contact stage (B), PM contact stage (C), and post-fusion stage (D). Blue arrows point to fusion pores; yellow arrows point to unresolved regions at the PM. Scale bars, $100 \mathrm{~nm}$. 


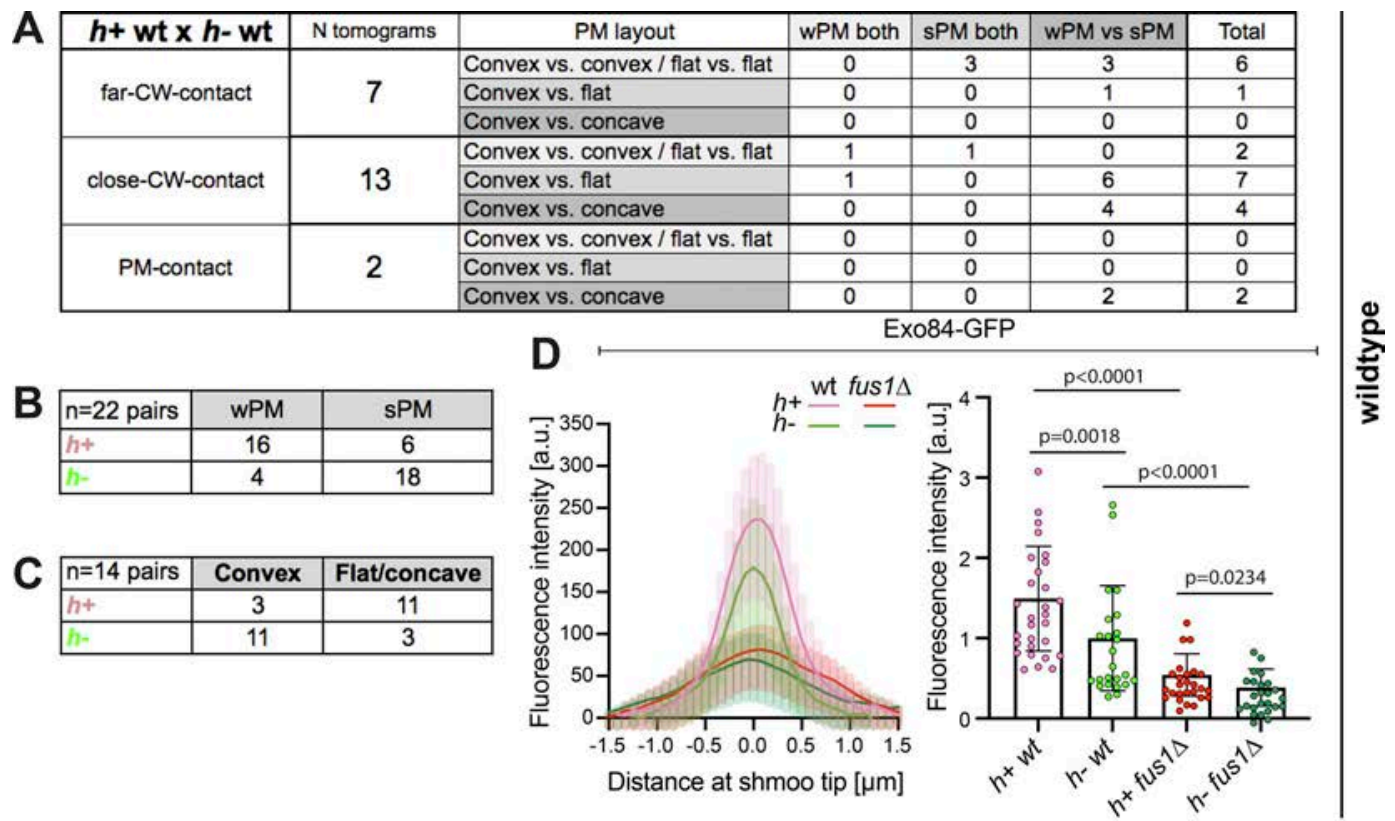

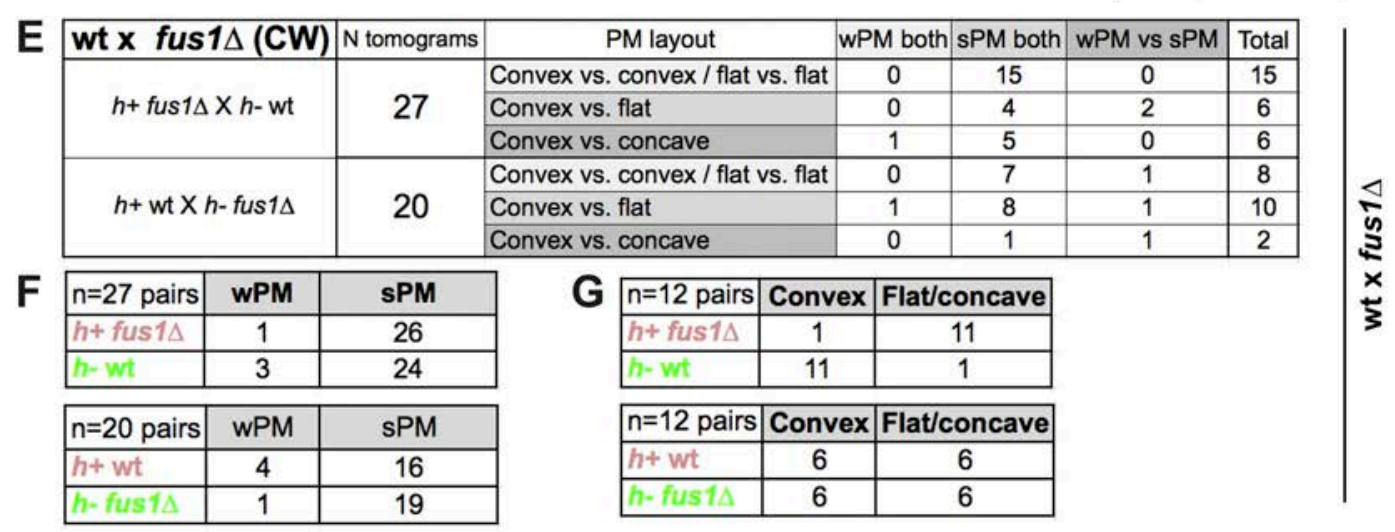

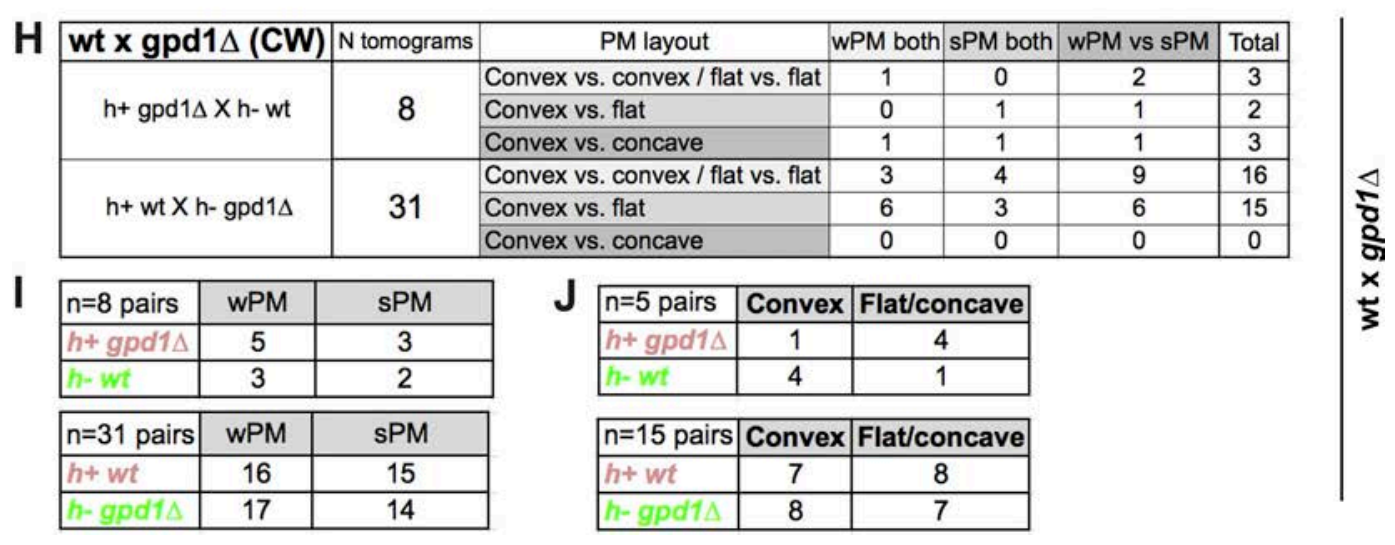

Figure S4. Classification of tomograms from heterothallic crosses and additional data on distribution of exocytic vesicles. (A) Table classifying the set of tomograms from WT $h+x h$ - cell pairs used in this study, according to PM morphology. (B) Table showing the number of each mating type in WT $h+x h-$ crosses with wPM or SPM. (C) Table showing, among asymmetric WT mating pairs, the number of each mating type with convex or flat/concave shmoo tip conformation. (D) Left: Fluorescence profile of Exo84 (GFP) along the shmoo tips of labeled WT and fus1 $1 \Delta$ cells crossed to unlabeled WT cells. Images were obtained from average projections of time-lapse videos acquired at a maximum speed during $3 \min (n=16 h+$ WT cells, $13 h-W T$ cells, $15 h+$ fus $1 \Delta$ cells, and $14 \mathrm{~h}$ - fus1 $\Delta$ cells). Right: Exo84 mean fluorescence intensity of the 5 maximum pixels around peak from profiles as on the left $(n=29 h+$ WT cells, $24 h-$ WT cells, $26 \mathrm{~h}+$ fus $1 \Delta$ cells and $25 \mathrm{~h}$ - fus $1 \Delta$ cells from two experiments; P values from Mann-Whitney tests). (E) Table classifying the set of tomograms from WT $\times$ fus $1 \Delta$ crosses used in this study, according to PM morphology in the CW contact stage. (F) Tables showing the number of each mating type in WT $\times$ fus $1 \Delta$ crosses with wPM or SPM. (G) Tables showing, among asymmetric WT $\times$ fus $1 \Delta$ mating pairs, the number of each mating type with convex or flat/concave shmoo tip conformation. (H) Table classifying the set of tomograms from WT $\times$ gpd1 $1 \Delta$ crosses used in this study, according to PM morphology in the CW contact stage. (I) Tables showing the number of each mating type in WT $\times$ gpd1 $1 \Delta$ crosses with wPM or sPM. (J) Tables showing, among WT $\times$ gpd $1 \Delta$ asymmetric mating pairs, the number of each mating type with convex or flat/concave shmoo tip conformation. 
A

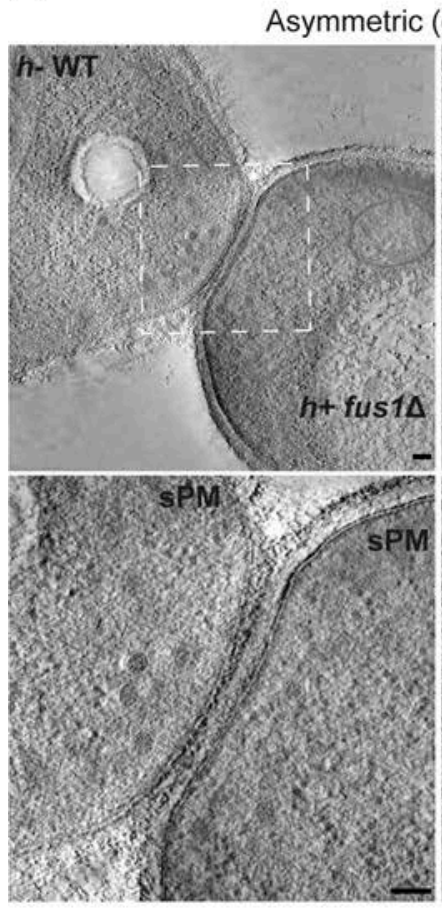

$h-\mathrm{WT} \times h+$ fus1 $1 \Delta$

Asymmetric (convex / concave)

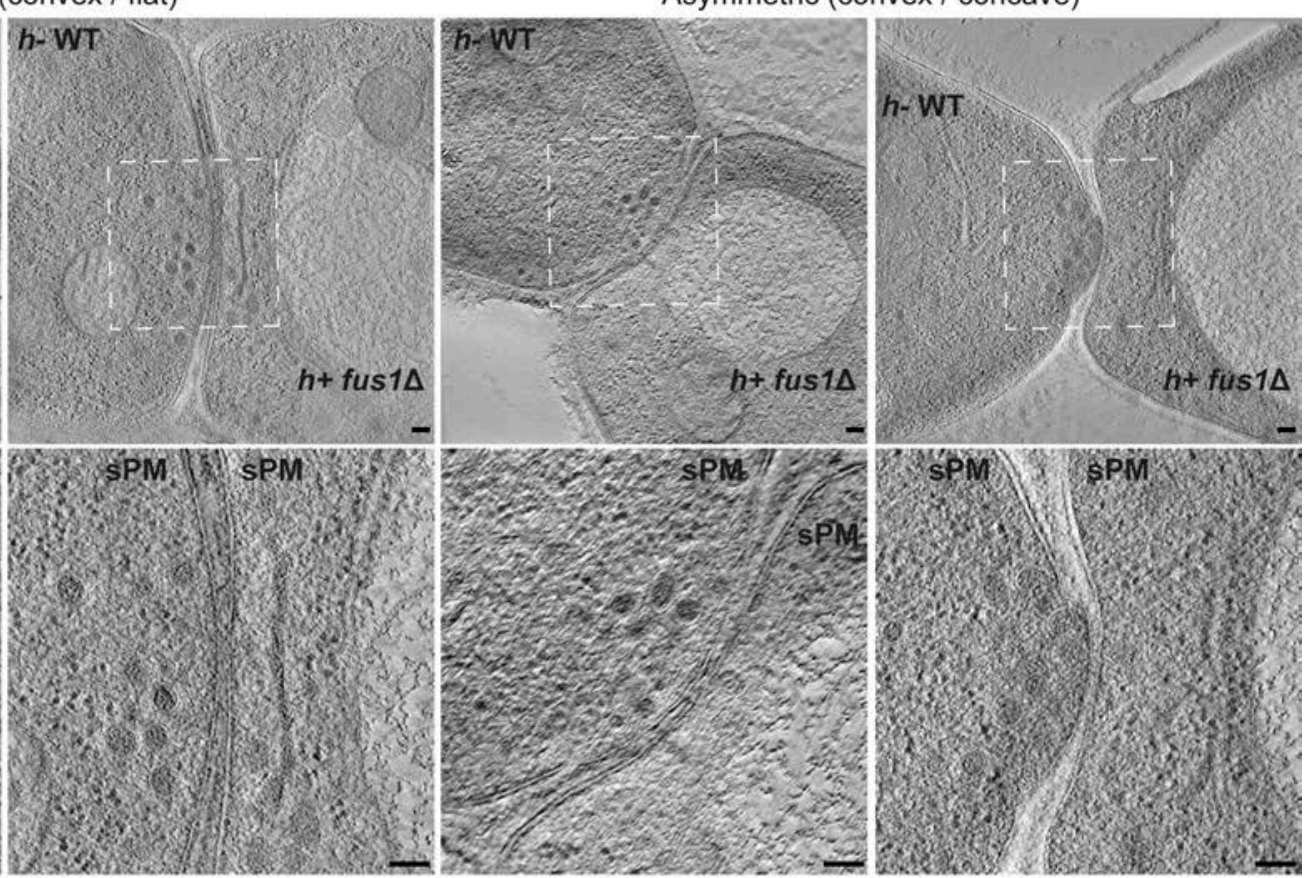

B

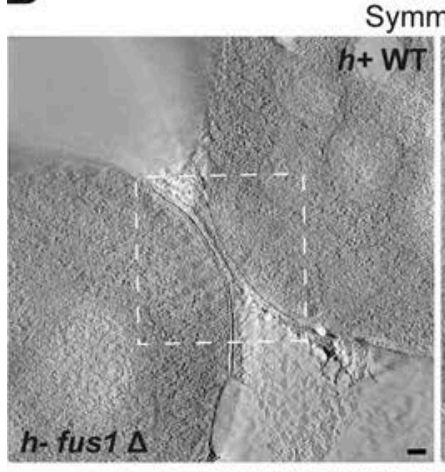

Symmetric

$h-f u s 1 \Delta \times h+W T$
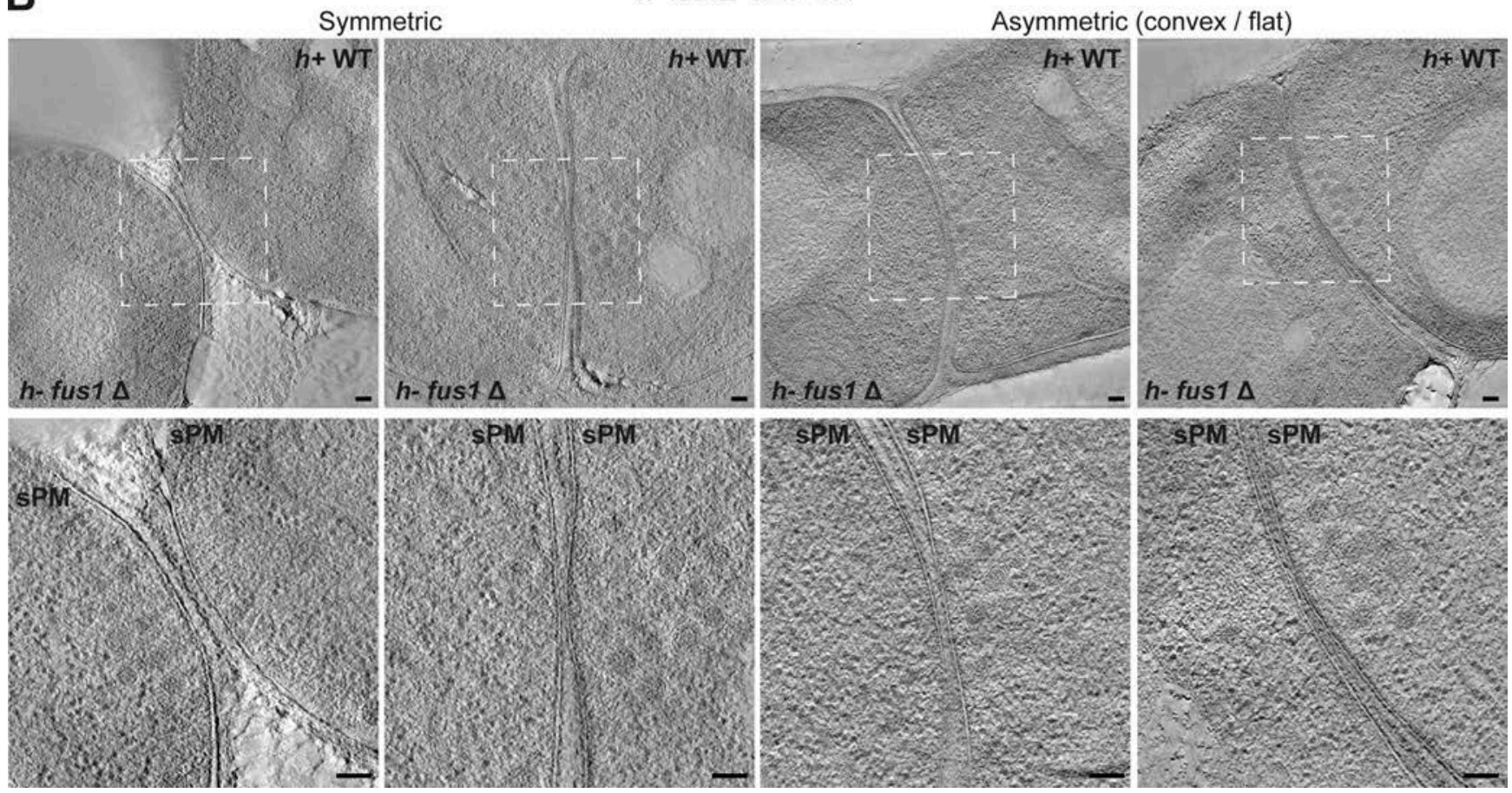

Figure S5. Morphology of WT $\times$ fus1 $1 \Delta$ crosses. Virtual z-slices through tomograms of mating cells in CW contact stage for $h+f$ fus $1 \Delta \times h-W T(A)$ and $h-f$ us $1 \Delta$ $\times h+$ WT (B). Scale bars, $100 \mathrm{~nm}$.

Video 1. Tomogram and model of a cell pair prefusion. The video shows all virtual z-sections of the tomographic reconstruction of a prefusion site at the close CW contact stage. The PM is segmented in cyan, vesicles are shown in pink (large vesicles) and purple (small vesicles), and filaments are segmented with a yellow line. The left cell shows a convex shape with a sPM. The cell on the right shows a slightly concave shape at the site of cell-cell contact with PM that exhibits an undulating pattern. Scale bar, $100 \mathrm{~nm}$. 
Video 2. Tomogram and model of a cell pair after fusion. The video shows all virtual z-sections of the tomographic reconstruction of a cell pair after fusion. The PM is segmented in cyan, vesicles are shown in pink (large vesicles) and purple (small vesicles), and filaments are segmented with a yellow line. Scale bar, $100 \mathrm{~nm}$.

Video 3. Tomogram and model of a cell pair with several connections. The video shows all virtual z-sections and PM segmentation (green) of the tomographic reconstruction of a cell pair exhibiting at least two apparent connections between cells. This corresponds to the example shown in Fig. $3 \mathrm{C}$ and Fig. S2 A. Scale bar, $100 \mathrm{~nm}$.

Video 4. Tomogram and model of a cell pair with an asymmetric pore. The video shows all virtual z-sections and PM segmentation (green) of the tomographic reconstruction of a cell pair with a noncircular pore. This corresponds to the example shown in Fig. 3 D and Fig. S2 B. Scale bar, $100 \mathrm{~nm}$.

Video 5. Tomogram and model of a cell pair with an asymmetric pore. The video shows all virtual z-sections and PM segmentation (green) of the tomographic reconstruction of a cell pair with a noncircular pore. This corresponds to the example shown in Fig. S2 D. Scale bar, $100 \mathrm{~nm}$.

Video 6. Tomogram and model of a cell pair with a pore containing a strand of $\mathbf{C W}$. The video shows all virtual z-sections and PM segmentation (green) of the tomographic reconstruction of a cell pair whose fusion pore is split in two by a strand of CW. This corresponds to the example shown in Fig. $3 \mathrm{E}$ and Fig. S2 C. Scale bar, $100 \mathrm{~nm}$.

Table S1 is provided as a separate file online and lists yeast strains used in this study. 